\title{
Distributed Clustering Approach for UAV Integrated Wireless Sensor Networks
}

\author{
Huseyin Okcua, Mujdat Soyturk ${ }^{b,{ }^{*}}$ \\ a Naval Science and Engineering Institute, Tuzla, Istanbul, 93942 - Turkey \\ ${ }^{b}$ Department of Computer Engineering, Marmara University, Kadikoy, Istanbul 34722, Turkey
}

\begin{abstract}
Wireless Sensor Networks consist of battery-limited sensor nodes which have the ability of sensing the environment, communicating with other nodes and processing the data. Large number of sensor node deployment over a geographical area imposes some constraints on the retrieval of the data. The use of mobile sinks (e.g., Unmanned Aerial Vehicle, UAV) is an effective solution method for such large-scale networks. However, depending on the path and altitude of the UAV, and the type of radios in use, coverage problem arises where some nodes cannot get connected to the UAV. In this paper, the coverage problem is examined where UAV is used as mobile sink node. On the basis of our analysis, a dynamic and distributed clustering approach is proposed. Evaluations are performed with a realistic simulation environment. Performance results show that proposed approach reduces the energy-consumption and construct more stable and well balanced clusters that connect the uncovered nodes to the UAV.
\end{abstract}

Keywords: Wireless Sensor Networks (WSNs), Energy Efficiency, Network Lifetime, Clustering, Unmanned Aerial Vehicle (UAV), Received Signal Strength (RSS), Received Signal Strength Indicator (RSSI), Coverage Problem.

\footnotetext{
"Corresponding author. Mujdat Soyturk

E-mail addresses: mujdat.soyturk@marmara.edu.tr, hokcu@dho.edu.tr
}

Please note that this version of the paper is the post-peer review, accepted paper submitted for final publication. The printed and reformatted version is accessible at the original source of the publication (http://www.inderscience.com/info/inarticle.php?artid=59912), where cited as "Huseyin Okcu, Mujdat Soyturk. Distributed Clustering Approach for UAV Integrated Wireless Sensor Networks, International Journal of Ad Hoc and Ubiquitous Computing, Vol.15, No. 1-3, pp.106-120, 2014 (2014)". 


\section{Introduction}

Sensor networks are composed of large number of tiny, low power and low-cost sensor nodes that have the ability of sensing the environment, processing the data and communicating with other nodes. These nodes monitor and measure physical phenomena (e.g. humidity, temperature, hostile action, flood, vibration etc.) to be used in the application areas of agriculture, healthcare, border protection and security surveillance, logistics and transportation, disaster management and military. In these application areas, the use of wired sensors is not feasible and applicable due to large number sensors and inaccessible topological areas such as hostile and disaster fields [1,2]. Leaving these nodes unattended over inaccessible areas with a limited battery capacity makes power management highly critical issue in order to extend network lifetime. After the deployment phase, maximizing the sensor node's lifetime requires energy efficient architectures, algorithms, and protocols [3, 4].

Management and design of such unattended and large size networks would require scalable architectures and energy-efficient methods. Organizing nodes into groups called clusters has been very common method for network scalability and energy-efficiency [4]. Some set of nodes are chosen as Cluster Heads $(\mathrm{CH})$ which usually have more residual energy and proximity to the sink. Sensor nodes transmit their sensed data to the cluster head via multi-hop or single-hop transmission, then cluster heads aggregate data and send to a distant mobile or static sink.

Large number of sensor node deployment over a geographical area (e.g. sea, harsh terrain, and inaccessible areas such as hostile territories) imposes some constraints on data transfer to the sink. The use of static sinks is not practical in such application areas for maintaining connectivity due to limited transmission range of sensor nodes. Single-hop communication directly from nodes to sink is impossible due to long distances between nodes and is not feasible due to energy-efficiency reasons. On the other hand, the use of multi-hop communication to reach the sink node exhausts the battery of the nearest nodes to the sink earlier than others. Multi-hop communication also reduces end-to-end reliability due to network size and unreliable link conditions. Connectivity problems caused by random node deployment, noisy channels, harsh environments and link failures exacerbate this problem. Data acquisition via mobile sinks (e.g. UAVs, robots, submarines etc.) is an effective solution method for the retrieval of sensor data in inaccessible locations (hostile locations, disaster territories). In this approach, a mobile sink node moves over a sensor network, retrieves data from sensors within its radio range and maintains wireless connectivity for disconnected nodes. Data retrieving by mobile sink reduces energy consumption at nodes by reducing multi-hop communication, avoiding long distance transmissions and redundant transmissions $[5,6]$.

In this paper, we focus on RSSI-based clustering in Wireless Sensor Networks (WSNs) which use Unmanned Aerial Vehicles (UAVs) as mobile sinks. We first study the coverage problem in WSNs where a UAV is a mobile sink. We point out that depending on the path and altitude of the UAV and the transmission range of the radios used in communication, connectivity problems arise where many nodes remain uncovered. Rescheduling the UAV's flight path to cover each node in WSN is costly for different aspects. First, a path that will cover each node will extend the operation (flight) duration of the UAV where UAV may not endure to that extend. Longer paths or longer flight schedules will delay the acquisition of the overall data from the interest area. The worse is that longer paths cause more energy consumption in the WSN due to increase in the number of transmissions and receptions within the network. Based on our analysis, we propose a new clustering approach which uses received signal strength indicator (RSSI) 
values received from cluster head nodes and mobile sink node (UAV). In the proposed approach, we aim to reduce the energy consumption and interference at nodes, constructing more balanced and stable clusters with the selection of cluster heads considering the UAV path. We analyzed both the clustering phase and data gathering phase. Proposed clustering approach consumes less energy in both phases, which also constructs clusters in shorter time. Main difference of the proposed approach from the existing studies in the literature [4,11-19] is the parameters used in clustering approach. Studies in the literature generally use node degree, neighbor RSSI values as clustering parameters or probabilistic methods, whereas our approach uses RSSI values of UAV and the energy level at nodes in the cluster head selection. Clusters are constructed based on RSSI values and the energy levels at nodes. RSSI-based clustering and cluster head selection also allows nodes to manage power levels on transmissions to reduce the energy consumption and interference.

In order to obtain more accurate results, we use the IEEE 802.15.4 standard [7] compliant nodes and the IEEE 802.15.4 [7] compliant TI CC2420 [8] Network Interface Card (NIC) model. The standard, IEEE Std 802.15.4 [7], is defined in order to extend network lifetime in Wireless Personal Area Networks (WPANs), which comprises the physical layer (PHY) and medium access control (MAC) sublayer specifications of the low-data-rate wireless connectivity with fixed, portable, and moving devices with no battery or very limited battery consumption requirements. This standard provides compatible interconnection for wireless sensor nodes using low-data-rate, low-power, and low-complexity short-range radio frequency $(\mathrm{RF})$ transmissions in a wireless sensor network application area [7].

In order to keep the fidelity in the simulation of the system and in the evaluations of the proposed approach, we use the models and parameters defined in these standards. For the simulation environment OMNET++ with MIXIM Framework [31-33] is used. This framework provides IEEE 802.15.4 compliant TI CC2420 RF Transceiver model. In our simulations, node parameters such as Carrier Sense Multiple Access (CSMA) backoff values, transmit power levels, transmit/receive/sleep energy consumptions, are obtained from TI CC2420 Datasheet [8].

The work in this paper is one of the first studies that examine the UAV coverage on WSNs. In particular, our contributions are as follows:

- We point out that some nodes within the network may remain uncovered depending on the path and altitude of the UAV. Due to the low-cost sensors which operate with low-data-rate, short range radios, the UAV has to fly at an altitude within sensor range. Therefore, some nodes remain uncovered depending on the path and altitude of the UAV.

- Our analysis suggests that clustering in such environments is a feasible approach to increase the coverage. Uncovered nodes can get attached to the covered nodes in vicinity which thereafter construct a cluster. The important issue is the selection of cluster heads considering the path of the UAV.

- Our analysis also suggests that depending on the selected cluster heads, energy consumption on communications between the UAV and cluster head can be reduced while the energy consumption on communications between sensor nodes and cluster head can also be reduced.

- We present a clustering approach to reduce the energy consumption on clustering and communications to extend the lifetime of the network. Proposed approach uses the RSSI values received from UAV and the remaining energy levels of the nodes for the selection of cluster head. Member nodes of the clusters are also selected 
considering the RSSI values of the cluster heads. Proposed approach also provides more stable clusters where single node clusters are avoided.

This paper is organized as follows. In the second section, problems related with energy consumption and connectivity are defined for UAV integrated WSNs. Studies and related work on clustering approaches are presented in the third section. In the fourth section, UAV integrated heterogeneous network model is examined and the proposed clustering approach is presented. Simulation and results are presented in the fifth section, and finally, we conclude our discussion in the last section.

\section{Problem Statement}

In multi-hop communications in WSNs, nodes closer to a stationary sink node die earlier than others, introducing a connectivity problem. Mobile sink (e.g. UAV) has ability to move along a path to provide connectivity and helps balancing the load on nodes. In such an environment, each node has opportunity to access mobile sink node (UAV) while the mobile sink node is within its communication range.

In such a UAV integrated heterogonous architecture, however, defining the operation altitude of the UAV becomes a problem. Sensor nodes are usually disposable cheap nodes, and therefore they usually have limited resources. Communications between sensor to sensor nodes and between sensor nodes and UAV have to be conducted with the same type of interfaces at nodes. Although, UAVs have more capabilities, they use identical radios to communicate with sensor nodes. For this reason, UAVs have to fly over the operation area at an altitude which sensor nodes can be able to communicate with UAV. We examine the connectivity and the coverage of sensor network area for different UAV altitudes. In this experiment, a flat network topology is used to observe the uncovered and inaccessible nodes. Homogenous nodes are dispersed over $2000 \times 2000 \mathrm{~m}^{2}$ area uniformly. UAV flies with $20 \mathrm{~m} / \mathrm{s}$ speed and broadcasts beacon message for every two seconds. The operation altitude is bounded with 250 meters due to the use of IEEE 802.15.4 compliant TI CC2420 radio. TI CC2420 radio has 8 different power levels with $1 \mathrm{~mW}$ as the highest level. When the sensitivity of the radio is adjusted to $-95 \mathrm{dBm}$ as indicated in the datasheet [7] and the path loss exponent is assigned to 2.5 for open space environment, the maximum transmission distance without packet losses is found as 250 meters. Therefore, the operation altitude should be at most $250 \mathrm{~m}$ in order to setup connection between UAV and ground level nodes. In section 5, power levels and transmission distance are discussed in detail.

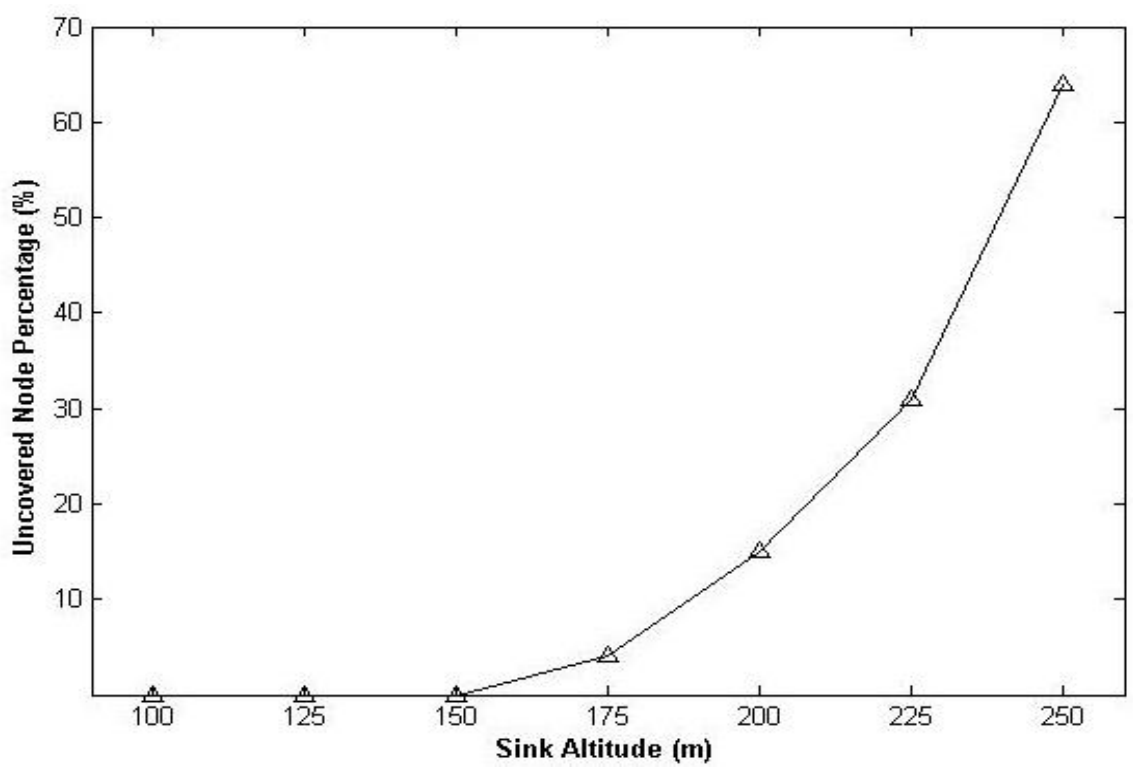

Figure 1 Effect of Sink Altitude on Coverage. 
Fig. 1 shows the coverage of UAV for different flight altitudes. It is seen that as the operation altitude of the UAV increases, more number of nodes get uncovered. In other words, connectivity of the nodes to the UAV decreases due to less number of nodes covered by the UAV. Moreover, coverage reduction increases exponentially. At the altitude of 250 meters, more than 150 of total 250 nodes become uncovered (63\% of total nodes) in a flat topology WSN where there is not any clustering. It means that some portions of the operation area will remain uncovered as the altitude increases. Uncovered nodes in this region will not be able to send their data to UAV. Fortunately, these uncovered nodes can use the covered nodes as relay nodes to send their data to the UAV. Therefore, clustering appears as a good solution for the connectivity problem where clusters are organized to cover uncovered nodes and to have connection with UAV to deliver sensed data to the UAV.

In addition to the problem given above, there is another well-known problem related with energy consumption on transmissions. Transmissions to distant destinations consume more power. As the distance between communicating nodes increases, energy consumption increases exponentially. Dissipated energy during the transmission $\left(E_{T x}\right)$ depends on the desired distance to be reached as indicated in Equation (1) [12];

$$
E_{T x}(k, d)=k \times E_{\text {elec }}+k \times E_{\text {amp }} \times d^{2}
$$

where $k$ is the number of the bits of packets, $E_{\text {elec }}$ is the energy dissipated in electronic circuits, $E_{a m p}$ is the energy dissipated for transmission in power amplifier and $d$ is the transmission distance.

In order to reduce the energy consumption on communications, we state that clusters can be organized considering the distance to UAV and distance to other neighbor nodes. Nodes closest to the UAV can be selected as Cluster Head $(\mathrm{CH})$. This approach will reduce the energy consumption significantly in case of the use of adaptive transmission power levels (as defined in TI CC2420 RF Transceiver Datasheet) and provides more robust communication approach between the sensor nodes and UAV. Moreover, this approach reduces the interference at nodes in the operation area. Transmission Equation (2) [20] shows that the interference distance is dependent on transmission power level.

$$
d=\left(\frac{P_{t x} \cdot \lambda}{16 \pi^{2} \cdot P_{r x}}\right)^{1 / \alpha}
$$

where $\alpha$ is the path loss exponent, $P_{t x}$ is the transmission power level and $P_{r x}$ is the reception power level.

Depending on the sensor type and the radio module, energy consumption values on transmissions and receptions vary. Energy consumptions for different sensor nodes and radio modules are summarized in [9]. It is seen that energy consumption on reception is as high as on transmissions. As defined in CC2420 radio [8], the receive (RX) current level value is $18.8 \mathrm{~mA}$ where the maximum transmit (TX) current level is $17.9 \mathrm{~mA}$, and in Telos mote [10] the RX current level value is $21.8 \mathrm{~mA}$ where maximum TX current level is $19.5 \mathrm{~mA}$. We conclude that the energy consumption on receiver node extremely increases as the interference gets higher. Therefore, we state that in order to reduce the energy consumption at nodes, nodes that have shorter distances to cluster members and shorter distance to UAV should be selected as cluster heads.

\section{Related Work}

There are a number of clustering algorithms proposed to enhance WSNs in different aspects $[4,11-19]$. Common main goal in these approaches is providing energy efficiency 
in terms of reducing the energy consumption and prolonging the lifetime (Table 1). Other goals include load balancing, fault tolerance, increasing connectivity, reducing end-to-end delay, and optimization of cluster count.

Table 1 Properties of Clustering Algorithms

\begin{tabular}{|l|c|c|c|l|}
\hline \multicolumn{1}{|c|}{ Algorithms } & Complexity & $\begin{array}{c}\text { Sink } \\
\text { Mobility }\end{array}$ & $\begin{array}{c}\text { Cluster } \\
\text { Topology }\end{array}$ & Clustering Purpose \\
\hline LEACH & $\mathrm{O}(1)$ & Static & Single-hop & Load balancing \\
\hline LEACH-C & $\mathrm{O}(1)$ & Static & Single-hop & $\begin{array}{l}\text { Load balancing/ } \\
\text { Energy efficiency }\end{array}$ \\
\hline EEHC & $\mathrm{O}(\mathrm{kh})$ & Static & Multi-hop & Energy efficiency \\
\hline HEED & $\mathrm{O}(1)$ & Static & Single-hop & $\begin{array}{l}\text { Load balancing/ } \\
\text { Energy efficiency }\end{array}$ \\
\hline ExHEED & $\mathrm{O}(1)$ & Static & Single-hop & $\begin{array}{l}\text { Load balancing/ } \\
\text { Energy efficiency/ } \\
\text { Cluster stability }\end{array}$ \\
\hline DWEHC & $\mathrm{O}(1)$ & Static & Multi-hop & $\begin{array}{l}\text { Energy Efficiency/ } \\
\text { Load balancing }\end{array}$ \\
\hline Fazackerley et al & $\mathrm{O}(1)$ & Static & Single-hop & Energy Efficiency \\
\hline Lotfinezhad and Liang & $\mathrm{O}(1)$ & Mobile & Single-hop & Energy Efficiency \\
\hline Pantziou et al & $\mathrm{O}(1)$ & Mobile & Multi-hop & $\begin{array}{l}\text { Energy Efficiency/ } \\
\text { Load balancing }\end{array}$ \\
\hline Blace et al & $\mathrm{O}(1)$ & Static & Single-hop & $\begin{array}{l}\text { Threat awarenes/ } \\
\text { Energy Efficiency }\end{array}$ \\
\hline
\end{tabular}

One of the early studies in the literature is Low-Energy Adaptive Clustering Hierarchy $(\mathrm{LEACH})$ [11]. In LEACH algorithm, at each clustering round, new $\mathrm{CHs}$ are selected by rotating cluster head role among all nodes in order to obtain load balancing. Cluster head selection is based on a probabilistic method which does not consider energy levels at nodes. Authors of the LEACH later, proposed another algorithm LEACH-C [12] which uses a centralized method to control clustering process by remote base station. LEACH-C aimed to select $\mathrm{CHs}$ with higher energy levels. Each node sends information about its current location and energy level to the base station (BS), in order to obtain load balancing and select $\mathrm{CHs}$ with the high energy level. Energy Efficient Hierarchical Clustering (EEHC) [13] algorithm forms multi-tier clustering topology that provides k-hops intra-cluster topology and h-hops connectivity between $\mathrm{CHs}$ to sink. Nodes send their sensed data from lower layer $\mathrm{CHs}$ to upper layer $\mathrm{CHs}$ respectively. The time complexity of this algorithm is $O\left(k_{1}+k_{2}+\ldots+k_{h}\right)$, which is a significant disadvantage compared to $O(1)$ complexity clustering algorithms such as LEACH. Hybrid Energy-Efficient Distributed Clustering (HEED) [4] is another distributed clustering algorithm that aims to select CHs among nodes which have more residual energy levels. It introduces a cost function to provide distributed $\mathrm{CH}$ selection. HEED has four main goals; prolonging network lifetime by distributing energy consumption, terminating the cluster head selection process within a certain number of iterations, minimizing control overhead, and producing well-distributed cluster heads with compact clusters. Although HEED is an efficient clustering algorithm, it has a problem related with the size of the clusters. In case of a decrease in the density of the network or the transmission range, some clusters are formed with no members but with a single cluster head, although these single cluster heads have connectivity to their neighbors. Its effects on the WSN are unbalanced cluster formation and increased energy consumption. Extended HEED (ExHEED) [14] algorithm which is an extended version of HEED algorithm, is proposed to reduce the energy consumption by reducing the number 
of CHs with the use of a core extraction algorithm [15]. It also helps to reduce the size of the routing table at nodes. In this algorithm, a set of node is selected as core nodes where only core nodes compete to become $\mathrm{CHs}$. Although ExHEED reduces the single $\mathrm{CH}$ count compared to HEED with the use of core extraction algorithm, it inherits the single cluster head problem of HEED. Moreover, core extraction algorithm introduces additional clustering delay and energy consumption. Distributed Weight-based Energy-efficient Hierarchical Clustering (DWEHC) [16], which is a weight based algorithm, is proposed to obtain more balanced clusters than HEED algorithm and to optimize the intra-cluster topology. The weight function is the product of the sensor's energy level and the proximity to the neighbors. DWEHC forms well-balanced clusters with its multi-hop intra cluster topology and has low energy consumption due to low cost transmission policy. In order to ensure low cost transmission, DWEHC calculates distances between nodes with the position information. Therefore, GPS-integrated sensor nodes are required for precise distance calculation, as it is assumed in [16]. Fazackerley et.al [17] proposed RSSI-based clustering algorithm to select cluster heads in the regions of high density. Each node competes to become a cluster head with the use of the RSSI values gathered from neighbors as promotion factor. This approach reduces the transmission link length and its variance for energy efficiency, but it does not consider the residual energy of nodes.

Lotfinezhad and Liang [19] proposed a clustering scheme for wireless sensor networks with reachback mobile agents (C-SENMA) that groups sensors into clusters such that nodes communicate only with the nearest $\mathrm{CH}$ and the $\mathrm{CH}$ takes the task of data aggregation and communication with the mobile agent (UAV). CHs use a low-overhead MAC mechanism very similar to the conventional ALOHA to contend for the channel. Pantziou et al. [18] proposed cluster structures with the use of mobile sink in order to ensure balanced energy consumption among network nodes and prolonged network lifetime. Rendezvous Nodes (RN) are used for accessing to the mobile sink in urban areas. They examined the efficiency of data gathering with public transportation vehicles as mobile sink which recurrently follow a predefined trajectory in periodic intervals. Residual energy and distance to the trajectory are used as parameters in selecting $\mathrm{CH}$ and RNs. Blace et al. [21] proposed clustering algorithm that considers threat which destroys the sensor nodes. Proposed $\mathrm{CH}$ selection approach is a derivation of HEED, where $\mathrm{CH}_{\text {Prob }}$ is contrarily calculated considering mobile threat trajectory. Nodes that are close to the threat have low probability of becoming a $\mathrm{CH}$. Thus, network longevity is ensured by keeping $\mathrm{CHs}$ far from mobile threat. Sugar and Imre [22] and Denko [23] proposed clustering methods that use software agents. These agents collect routing and clustering information and periodically maintain the corresponding tables. Routing mobile agents traverse the network to form and deliver routing tables. On the other hand, static agents at nodes decide to form a cluster, divide a cluster or merge two clusters.

Morsly et al [24] proposed a clustering algorithm for sensor node mounted UAVs in order to fulfill security monitoring of a given area. The area is divided into zones and UAVs operate on the corresponding assigned zone. At each zone, UAVs elects the UAV-head by proposed clustering algorithm. However, Multi-UAV architecture introduces problem of designing efficient network between UAVs [25].

In this paper, we propose a distributed clustering approach for WSNs where a single UAV is used as a mobile sink. Compared to the approaches in the literature, further aspects of the problem are considered in the formation of clusters and selection of cluster heads. The proposed approach considers nodes' energy levels, their proximity to mobile sink with the use of RSSI values, and RSSI values of neighbor nodes for cluster membership to form energy-efficient and balanced clusters. We aim to construct well-balanced clusters within the network and avoid the single cluster head problem. To the best of our knowledge, this 
study is one of the first studies that considers UAV path in the formation of clusters. Proposed approach reduces energy consumption in communications between $\mathrm{CHs}$ and UAV and in communications between $\mathrm{CHs}$ and member nodes. We evaluate the proposed approach with the use of realistic path loss, MAC and PHY layer models with the help of MIXIM Framework.

\section{Network Model and the Proposed Approach}

In this section, we first define the heterogeneous network model that will be used in the design of the system. Later, the clustering approach proposed for UAV integrated heterogeneous WSN is presented.

\subsection{UAV Integrated Heterogeneous Network Model}

In the proposed approach, all sensor nodes are assumed to be identical and have the same resource and functional capabilities. Such kind of networks can be considered as homogenous network. However, the UAV which is considered as the sink node has more functionalities and resource capabilities. UAV is able to move in 3 dimensions with variable speeds. It can carry more radios on board and has the capability to process and relay the data to an external node for further process. Moreover, UAV has intelligence and smartness to take its own decision or can be controlled and directed with the remote controllers. Therefore, we can say that the composed network is a heterogeneous network with the integration UAV.

In this network model, each node sends sensor data to its respective $\mathrm{CH}$. $\mathrm{CH}$, then, aggregates all data received from all cluster members. When the $\mathrm{CH}$ introduces the sink node (UAV), it sends aggregated data of the cluster to the mobile sink node as depicted in the Fig. 2.

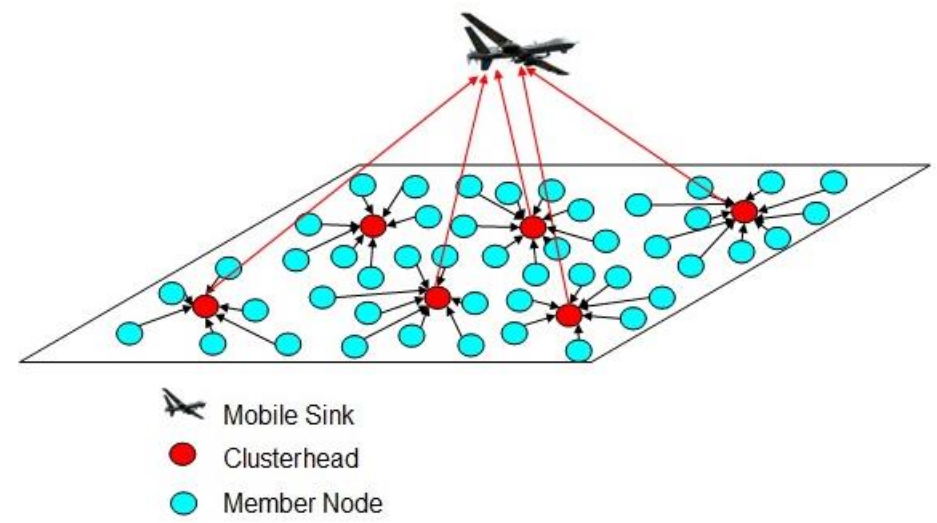

Figure 2 UAV Integrated Network Model

In the design of the proposed approach, following parameters and conditions are considered:

- Sensor nodes are randomly dispersed over the operation area.

- Sensor nodes in the network are stationary except UAV embedded node.

- Nodes are location-unaware, i.e. not equipped with GPS or localization service.

- All nodes have same resource capabilities (processing/communication/memory), and equal significance except UAV embedded node.

- $\quad$ Nodes are left unattended after deployment.

- The application area is inaccessible and harsh environment. 


\subsection{RSSI-based Hybrid and Energy-Efficient Distributed Clustering}

In order to solve the stated problems on coverage, connectivity and energy consumption, we propose a new clustering algorithm called RSSI-based Hybrid and Energy-Efficient Distributed Clustering (rHEED) which is based on the HEED algorithm. However, there are significant differences compared to HEED as described in the remaining part of this section. rHEED algorithm utilizes the RSSI values of the packets received from UAV and considers the remaining energy levels of nodes in the selection of CHs. It also utilizes the RSSI values to select member nodes in the formation of clusters.

Although HEED is an effective clustering algorithm it suffers some problems related to distributed $\mathrm{CH}$ selection. In the $\mathrm{CH}$ selection phase, some nodes declare itself as candidate $\mathrm{CHs}$ suppressing its neighbor nodes. In succeeding iterations, some of the candidates become $\mathrm{CHs}$ while the others back off due to more convenient candidate $\mathrm{CHs}$ nearby. The problem arises at this point. The neighbors (these are the suppressed nodes) of the backed off candidate nodes may or may not find another $\mathrm{CH}$ in their neighborhood. Nodes that do not find a candidate $\mathrm{CH}$ nearby remain unattended although they have connectivity with other nodes. At the end of the clustering algorithm of HEED, these unattended nodes declare themselves as $\mathrm{CHs}$ with no members. Moreover, these single $\mathrm{CHs}$ usually reside within the same area close to each other. There are many undesirable outcomes of this problem.

- Single CHs exist with no member in their own clusters.

- Single CHs increase the number of CHs.

- Number of CHs varies significantly without any control.

- Unequal size clusters are formed.

- Items above affect the energy efficiency and load balancing negatively in the WSN.

ExHEED aims to reduce the number of single $\mathrm{CHs}$ with the use of core extraction algorithm. Algorithm begins with core node selection process which allows only core nodes to become candidate CHs. The iterative phases of ExHEED to select CHs are exactly same as HEED, however, in ExHEED, only core nodes execute iterative phases. In the finalization phase, uncovered nodes run core extraction algorithm one more time to elect some extra CHs. By this way, ExHEED reduces the single $\mathrm{CH}$ count compared to HEED. However, there still remain some uncovered or unattended nodes which later become single cluster heads. Moreover, core extraction algorithm at the finalization phase introduces overhead and extends the clustering time to be the double of HEED. On the other hand, these two algorithms, HEED and ExHEED do not consider the position of the sink node.

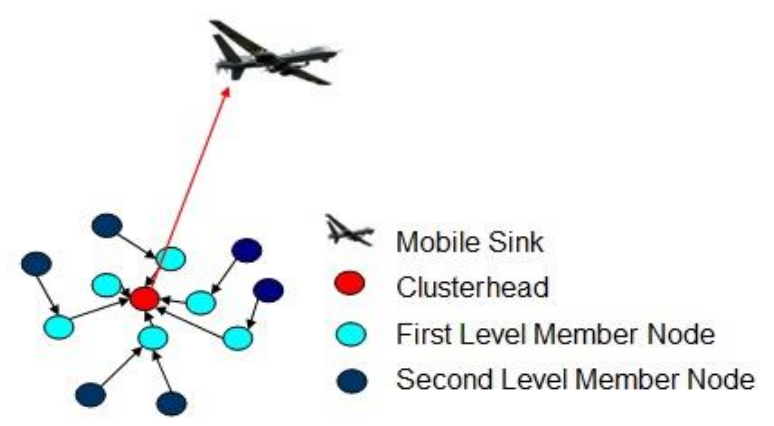

Figure 3 Cluster Formation in rHEED 


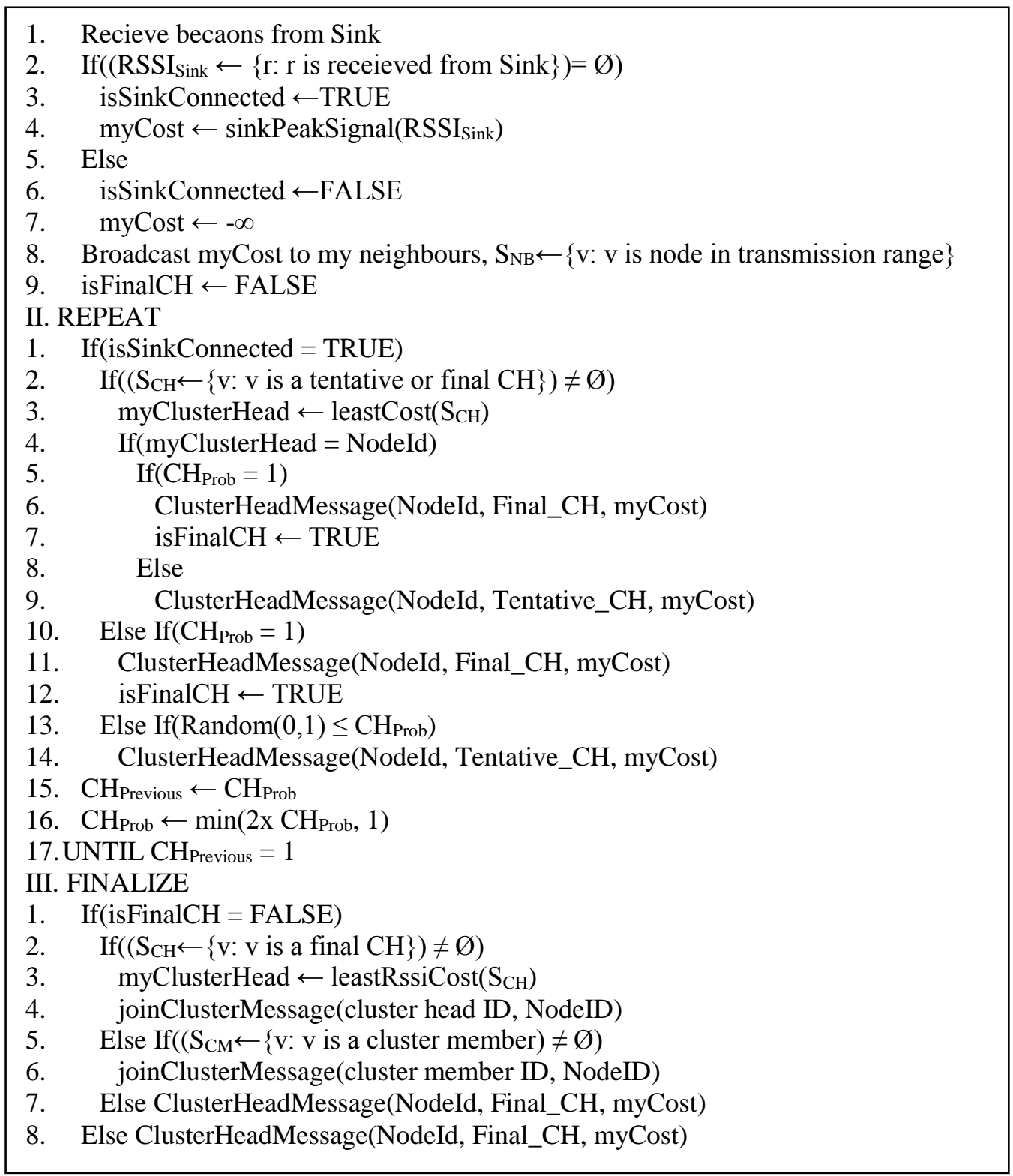

- Algorithm 1 Pseudo-Code of rHEED Algorithm

In the proposed approach, rHEED, cluster heads are selected considering both the residual energy levels of the nodes and their proximity to the mobile sink node (UAV). Proposed approach also eliminates the single $\mathrm{CHs}$ problem. Uncovered nodes after $\mathrm{CH}$ selection phase get connected to the nearest $\mathrm{CHs}$ with multi-hop paths if there are any neighbor nodes in vicinity (Fig. 3). Proposed approach allows nodes to use adaptable power levels for both intra-cluster and CH to UAV communications with the use of Link Quality Indicator (LQI) based distance calculation. The proposed approach has the following features:

- Clustering is completely distributed.

- Clustering terminates within a fixed number of iterations.

- At the end of each clustering process, each node is either a cluster head, or a cluster member (no unattended nodes remain).

- Clustering is efficient in terms of processing complexity and message exchange.

- $\mathrm{CHs}$ are relatively closer nodes to UAV.

- Only nodes those have UAV connection can be CHs. 
- Clusters are well-balanced with the use of RSSI values to form the clusters.

Proposed rHEED algorithm is presented in Algorithm 1. Sensor nodes record RSSI values of mobile sink (UAV) beacons in order to use these values in the clustering iterations. Only nodes that have UAV connection may become a $\mathrm{CH}$. Nodes can record more than one RSSI value of the UAV. In this case, the peak value of the RSSI recordings is used in the cost function (3) during the cluster head competition. On the other hand, each node also calculates the average RSSI value of UAV beacons. This value will be used by a $\mathrm{CH}$ to determine the time to send its packets to the UAV.

Algorithm starts with neighborhood discovery phase where calculated costs based on the $R S S I_{\text {peak }}$ are exchanged between neighbor nodes with the use of advertisement packets. Cost function is given as follows;

$$
\operatorname{Cost}_{i}=\max \left(R S S I_{i, \Phi}\right)
$$

where $i$ represents each sensor node in the WSN, and the $R S S I_{i, \Phi}$ is the received signal strength levels for node $i$ obtained from UAV beacons during its connection duration $\Phi$.

Neighborhood discovery phase is followed by a number of iterative steps to select the CHs between candidate nodes in a distributed manner. Each node that has a connection with UAV decides to become a cluster head by setting its probability of becoming cluster head, $\mathrm{CH}_{\text {prob }}$, as:

$$
C H_{\text {prob }}=\left\{\begin{array}{cl}
\max \left(C_{\text {prob }} x \frac{E_{\text {residual }}}{E_{\max }}, P_{\min }\right), & \text { if } \text { Cost }_{i}>0 \\
0, & \text { if } \text { Cost }_{i}=0
\end{array}\right.
$$

where $C_{\text {prob }}$ is the probabilistic value that limits the initial cluster head announcements, $P_{\min }$ is a small value that is used to limit the iteration number, and $E_{\max }$ is the highest power level of the node. Nodes with higher residual energy $\left(E_{\text {residual }}\right)$ and have connectivity with the UAV will have higher probability to become a $\mathrm{CH}$. We use $E_{\text {residual }} / E_{\max }$ ratio to calculate $\mathrm{CH}_{\text {prob }}$ similar to HEED, in order to support different energy levels in WSNs. This feature enables to use heterogeneous network elements/sensor nodes within the WSN.

During each iteration $1<i t e r<N_{i t e r}$, every node becomes a tentative $\mathrm{CH}$ with the probability of $\mathrm{CH}_{\text {prob }}$. This information is exchanged with neighbor nodes. Every node maintains a set of neighbor tentative $\mathrm{CHs}, S_{C H}$. A node $v_{i}$ selects its $\mathrm{CH}$ (my_cluster_head) to be the node with the least cost in its own $S_{C H}$. The least cost node may be itself among its neighbors. In this case, the probability $\mathrm{CH}_{\text {prob }}$ is doubled after each iteration. Until a tentative node's $\mathrm{CH}_{\text {prob }}$ reaches 1 , it broadcasts clusterHeadMessage(NodeId, Tentative_CH, myCost) to its neighbors. Nodes receiving clusterHeadMessage update their $S_{C H}$ in each iteration. If a tentative node's $\mathrm{CH}_{\text {prob }}$ reaches 1 , it finishes iterations phase with status set to Final_CH and broadcasts clusterHeadMessage(NodeId, Final_CH, myCost) to its neighbors. Nodes that are not elected as Tentative_CH or Final_ $\mathrm{CH}$ also finish iterations phase, if node's $\mathrm{CH}_{\text {prob }}$ reaches 1 . At the end of the iterations phase, nodes have status either Final_CH or normal node. In the finalization phase, normal nodes select their $\mathrm{CHs}$, if they have connectivity with a Final_CH status node, else nodes consider themselves uncovered. Uncovered nodes associate with the nearest cluster member node to become a second level member in that cluster.

When $P_{\min }$ is set to 0.005 , the algorithm is finalized at most 12 iterations. If $C_{\text {prob }}$ is set to 0.05 (that means $5 \%$ of the nodes will be $\mathrm{CHs}$ ), algorithm is finalized at least 6 iterations, because $\mathrm{CH}_{\text {prob }}$ converges to 1 after 6 iterations. Therefore, algorithm ends after constant number of iterations and has $O(1)$ complexity. 
In the finalization phase of the clustering algorithm, if there remain some uncovered nodes due to the problem of distributed approach, these uncovered nodes associate to the nearest cluster members as a second level cluster members. Therefore, clusters have maximum 2-hop distant members from CHs as shown in Fig. 3. In HEED algorithm, uncovered nodes are declared as $\mathrm{CHs}$ which leads to unbalanced network. In the proposed approach rHEED, clusters are more balanced compared to HEED and ExHEED. Moreover, selecting the nearest node to associate a cluster will reduce energy consumption due to the closer ranges of the nodes. Association delay is also much lower than the core extraction algorithm of ExHEED. One more benefit of the proposed approach, rHEED, is related with the interference caused on communications. Single cluster heads use high level transmission power to reach UAV which therefore cause high interference on neighboring nodes. This feature causes high energy consumption at neighbor nodes due to the RX currents on the receiver. With the use of rHEED, single cluster heads are avoided if the node has at least one neighboring node. Therefore, rHEED reduces the energy consumption on receptions and reduces interference at nodes.

\section{Simulations and Results}

We use OMNET++ [31] with MIXIM Framework [32, 33] to simulate the environment and test the proposed approach. MIXIM Framework has many features that are essential for wireless communications including wireless sensor networks, body area networks, ad-hoc networks, and vehicular networks. MIXIM provides and supports detailed models of wireless channel, radio wave propagation, interference estimation, radio transceiver power consumption, wireless connectivity, wireless MAC protocols, and other wireless protocols. Especially for WSNs, MIXIM provides IEEE 802.15.4 compliant TI CC2420 NIC model. TI CC2420 NIC is composed of CSMA/CA MAC and 802.15.4 PHY, which use original parameters defined in TI CC2420 datasheet such as current/power levels, transition periods, sensitivity etc. On the other hand, OMNET++ with MIXIM has mobility modules that support 2D and 3D mobility patterns which are essential for the test of the proposed approaches in this study.

\subsection{Sensor Node Model}

In the design and the evaluation of the proposed approach, we use IEEE 802.15.4 compliant TI CC2420 NIC model (see Fig. 4 (a)) developed by MIXIM Framework [32,33] developers totally adhered to TI CC2420 Transceiver datasheet [8]. In the MAC layer, CSMA-CA mechanism is used. On transmissions, output power levels (see Table 2) can be controlled considering the receiver. IEEE 802.15.4 CSMA mechanism is same as the IEEE 802.11 CSMA except Request to Send/Clear to Send (RTS/CTS) handshake mechanism. In order to avoid communication overhead introduced on broadcast communications with RTS/CTS mechanism in the large scale WSNs, IEEE 802.15.4 CSMA mechanism does not use RTS/CTS.

Table 2 CC2420 Transceiver TX Power Levels and Current Consumptions [8]

\begin{tabular}{|c|c|c|c|}
\hline $\begin{array}{c}\text { Output Power } \\
(\mathbf{d B m})\end{array}$ & $\begin{array}{c}\text { Output Power } \\
(\mathbf{m W})\end{array}$ & $\begin{array}{c}\text { Current Consumption } \\
(\mathbf{m A})\end{array}$ & $\begin{array}{c}\text { Transmission Distance } \\
(\mathbf{m})\end{array}$ \\
\hline \hline 0 & 1 & 17.4 & 250 \\
\hline-1 & 0.791 & 16.5 & 228 \\
\hline-3 & 0.501 & 15.2 & 189 \\
\hline-5 & 0.316 & 13.9 & 158 \\
\hline-7 & 0.199 & 12.5 & 131 \\
\hline-10 & 0.1 & 11.2 & 100 \\
\hline-15 & 0.0316 & 9.9 & 63 \\
\hline-25 & 0.00316 & 8.5 & 25 \\
\hline
\end{tabular}


TI CC2420 NIC model uses 802.15.4 compliant radio. It has advantages on managing transmission power for energy efficiency. In the PHY layer, sensor nodes can set TX current level to the power level determined by MAC, in order to drain minimum required energy from the battery of the sensor node. In Fig. 4 (a), TI CC2420 NIC model provided from MIXIM framework is presented. This NIC is used in our sensor node model (see Fig. 4 (b)) in order to fulfill PHY and MAC tasks defined in IEEE 802.15.4 such as LQI calculating, sleeping, transmitting, receiving etc. Proposed clustering approach is implemented in the network layer which interacts with the NIC to get required information such as RSSI values, receiver energy value from LQI, etc. In order to control TX power and TX current values, control messages are used between PHY and MAC layers to NETW layer (or vice versa) of the node without violating the CSMA-CA MAC and the 802.15.4 radio PHY semantics.

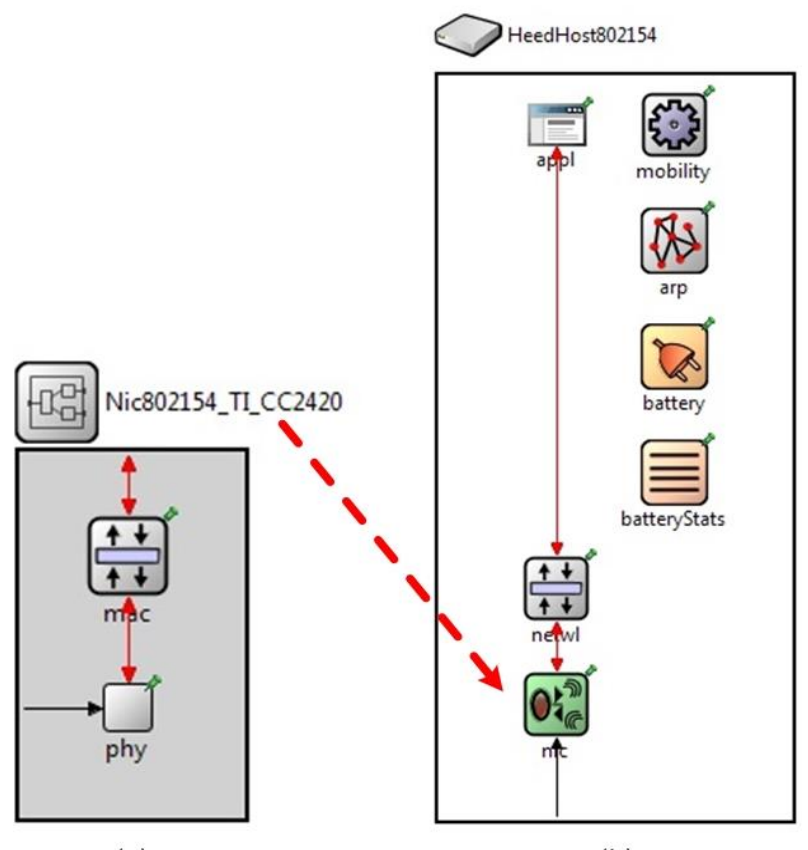

(a)

(b)

Figure 4 (a) TI CC2420 NIC Model. (b) 802.15.4 Compliant Node model with TI CC2420 NIC.

\subsection{Mobile Sink (UAV) Model}

UAV as the mobile sink node uses the identical radio (802.15.4 compliant radio) to communicate with the sensor nodes. However, UAV has more capabilities and functionalities compared to sensor nodes. These features can vary and depends on the type of the UAV. As defined in Section 2, the operation altitude of the UAV is bounded with 250 meters due to the use of IEEE 802.15.4 compliant TI CC2420 radio. On the other hand, the UAV has to fly at a speed to communicate with the sensor nodes along the path without losing any data sent from sensor nodes in the ground-to-air communication and also to make the each sensor node along the path receive the beacon messages of the UAV in the air-to-ground communication. There are several kinds of UAVs in the literature for different purposes with variable parameters and functions such as operation altitude, endurance, payload etc. Among the UAVs in the literature, mini-UAVs that meet the required characteristics in this study are presented in Table 3. Considering the operation area and the operation time in the air, Bayraktar [28] and the Falco [30] UAV models are the most appropriate vehicles for the proposed network model. 
Table 3 Mini-UAV Models.

\begin{tabular}{|l|c|c|c|c|c|}
\hline Characteristics & $\begin{array}{c}\text { WASP } \\
{[\mathbf{2 6}]}\end{array}$ & $\begin{array}{c}\text { RAVEN } \\
{[\mathbf{2 7}]}\end{array}$ & $\begin{array}{c}\text { BAYRAKTAR } \\
{[\mathbf{2 8}]}\end{array}$ & $\begin{array}{c}\text { PUMA } \\
{[\mathbf{2 9}]}\end{array}$ & $\begin{array}{c}\text { FALCO } \\
{[\mathbf{3 0}]}\end{array}$ \\
\hline Speed (m/s) & $11,1-18$ & $8,5-22$ & 17 & $11-23$ & 17 \\
\hline $\begin{array}{l}\text { Operation } \\
\text { Altitude (m) }\end{array}$ & $15-300$ & $30-150$ & 4,000 (ceil.) & 150 & $\begin{array}{c}5,000 \\
\text { (ceil.) }\end{array}$ \\
\hline Endurance (min) & 45 & $60-90$ & $60-80$ & 120 & $480-840$ \\
\hline Range (m) & 5,000 LOS & $8,000-12,000$ & $15,000-25.000$ & 15,000 & 200,000 \\
\hline
\end{tabular}

Mobility pattern of the UAV in the operation area is shown in Fig. 5. UAV sweeps the application area in straight lines from left-to-right until it reaches to border. It then makes up-to-down flight to take its position to sweep with another straight line from right-to-left. UAV follows similar legs until it sweeps the whole operation area. This flight schedule is considered as one round of UAV.

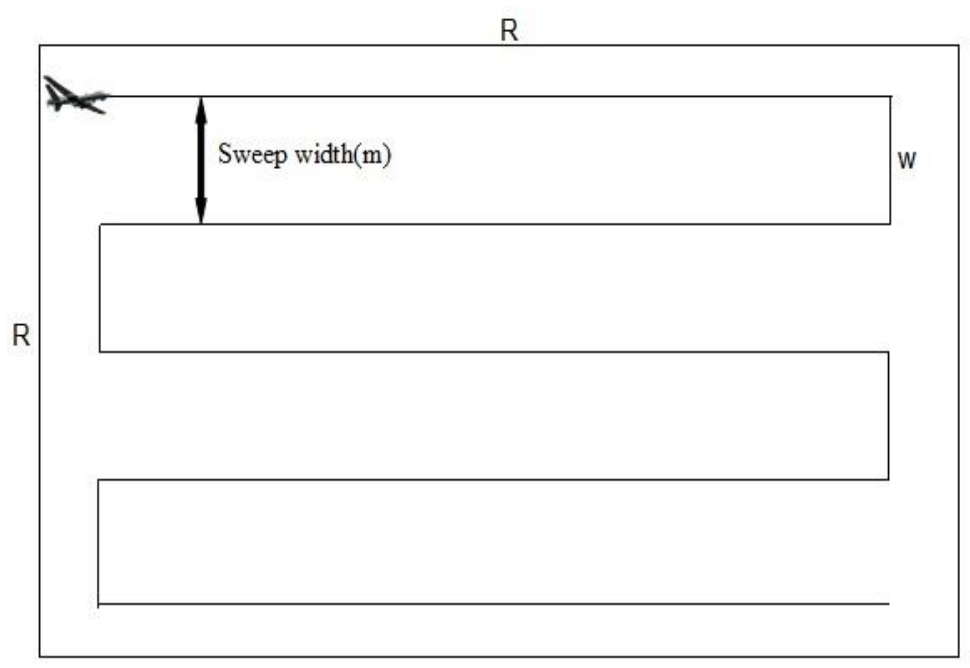

Figure 5 Movement Pattern of Mobile Sink

While the UAV takes its route, it sends beacon messages for every 2 seconds. Sensor nodes in the area record the RSSI values of the received beacons to use these values in clustering. First round is a blind run to allow sensor nodes learn the flight path of the UAV. After the first round, nodes calculate an average RSSI value;

$$
R_{S S I} I_{\text {avg }}=\left\{\begin{array}{cl}
\frac{\sum_{i=1}^{n} R S S I_{i}}{n,}, & \text { if } n>0 \\
0, & \text { if } n=0
\end{array}\right.
$$

Clustering phase is completed before the UAV sweeps the operation area at each round. In the sweep phase, the UAV collects the data from $\mathrm{CHs}$ while it also sends beacon messages. $\mathrm{CH}$ node only sends its aggregated data to the UAV, if only the RSSI value of the received beacons is higher than $R S S I_{\text {avg }}$ given in (5). Successive rounds continue with clustering and data gathering in turn. Order of the phases is presented in Fig. 6.

After the formation of the clusters, nodes adjust their TX power level according to the distance to their respective cluster head. If a node is cluster head then it adjust its TX power level with respect to the UAV. For the range calculation, nodes use LQI which is introduced by IEEE 802.15.4 standard. LQI measures the strength and/or quality of each received packet with using the receiver Energy Detection (ED) or a signal-to-noise ratio (SNR) estimation or combination of those [7]. LQI value is calculated by PHY layer than reported 
to the MAC layer for the usage in the upper layers. LQI value is more accurate than RSSI, because RSSI includes noise and signal where LQI includes only more accurate received signal strength value. Therefore, with the use of LQI value, nodes calculate more precise and accurate distance from neighboring nodes or UAV. This feature allows nodes to adjust TX power level according to calculated distance.

First Round:

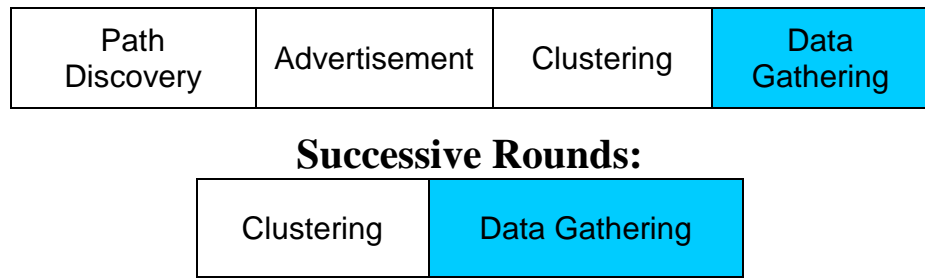

Figure 6 Phases of the Application of the rHEED Approach in Test Environment

We note that although nodes measure accurate distances to their neighbors, power levels that are defined in [8] are used. There are transmission power levels for different distance scales. Nodes select the appropriate one to make the receiver receive the signal with high quality. For example, for a measured distance $165 \mathrm{~m}$, the transmitting node has to use $0.316 \mathrm{~mW}$ output power which provides high quality signal at the receiver at most $189 \mathrm{~m}$ distance. Power levels defined in [8] are summarized in Table 2.

\subsection{Node Deployment}

Sensor nodes are randomly scattered into the $2000 \mathrm{~m} \times 2000 \mathrm{~m}$ operation area. In the experiments, mobile sink node (UAV) moves over the operation area with parallel scans to cover whole region as shown Fig. 5. Speed of the UAV is kept at $20 \mathrm{~m} / \mathrm{s}$ constant that it scans the whole operation area in 480 seconds. Traversing the whole operation area is considered as one tour. At the end of one tour on the field, mobile sink takes its initial position. Sensor nodes in the operation area records the RSSI levels of the beacons received from the UAV, so sensor nodes use this information at the end of each tour for clustering. Clustering phase at nodes is followed by the data gathering phase. When the UAV follows its path over the operation area, $\mathrm{CH}$ nodes send their collected/buffered data to the UAV (sink).

Compliant to the TI CC2420 NIC model, maximum 250m transmission range is used for both intra-cluster communication and $\mathrm{CH}$ to UAV communication. In the open space network field, simple path loss model is applied and path loss exponent is set to 2.5 and nodes receiver sensitivity is set to $-95 \mathrm{dBm}$. Although free space path loss model is used in simulations, obstacles and weather conditions can affect the path loss and can increase the path loss exponent.

Results are average values of data gathered from 10 different runs with different node positions and random numbers generated by different seeds at each run.

Table 4 Simulation Parameters

\begin{tabular}{|l|l|}
\hline \multicolumn{1}{|c|}{ Parameter } & \multicolumn{1}{c|}{ Value } \\
\hline \hline Simulation Area & $2000 \mathrm{~m}$ x 2000m \\
\hline Number of Sensor Nodes & 250 nodes \\
\hline Max. Transmission Range & $250 \mathrm{~m}$ \\
\hline Simulation Duration & $11000 \mathrm{sec}$ \\
\hline Sink Altitude & $150 \mathrm{~m}, 175 \mathrm{~m}, 200 \mathrm{~m}, 225 \mathrm{~m}$ \\
\hline Sink Velocity & $20 \mathrm{~m} / \mathrm{sec}$ \\
\hline Sink Beacon Period & $2 \mathrm{sec}$ \\
\hline Data Gathering Period & $960 \mathrm{sec}$ \\
\hline
\end{tabular}


Sink altitude affects the connectivity and energy consumption of the network. For this reason, simulation measurements are observed at different mobile sink altitudes, at $150 \mathrm{~m}$, $175 \mathrm{~m}, 200 \mathrm{~m}$ and $225 \mathrm{~m}$ as shown in Table 4.

\subsection{Compared Protocols}

Proposed approach has been compared with HEED and ExHEED algorithms. HEED algorithm is considered as a benchmark algorithm, where ExHEED algorithm makes improvements on HEED for various metrics. Moreover, these two algorithms have superiority in terms of energy efficiency and load balancing compared to other energy-efficient algorithms [11,12,13].

In performance evaluations, in addition to the canonical metrics such as energy consumption and network lifetime, some additional metrics such as number of $\mathrm{CHs}$, average distance to $\mathrm{CHs}$ are observed. Energy related performance metrics are the energy consumption on clustering phases, energy consumption on network operations, and total energy consumption.

Mobile sink altitude has effects on data gathering and connectivity. For this reason, energy consumption on data gathering period and node coverage parameters at different mobile sink altitudes are evaluated in the simulation. We examined and compared rHEED protocol with HEED and ExHEED protocols to show superiority and drawback of the proposed approach.

\subsection{The Effect of Sink Altitude}

Sink node's altitude has great effect on the coverage and on the success of the deployed system on the operation area. Sink node's altitude should be within the communication range of the communicating sensor nodes. We present the effects of variable UAV altitude on coverage. As shown previously in Fig. 1, as the UAV's altitude increases, the coverage of UAV over the network reduces. For the altitudes lower than 150, nodes can access to UAV directly. However, this operation altitude is not a feasible for many application areas. Operation terrain and the risk of management do not allow UAVs to operate at lower altitudes. For the altitudes higher than $150 \mathrm{~m}$, coverage and connectivity problems arise. And, for the $250 \mathrm{~m}$ altitude (maximum transmission range with the given parameters for TI CC2420 radio), uncovered nodes arise to a value of $63 \%$ of total nodes.

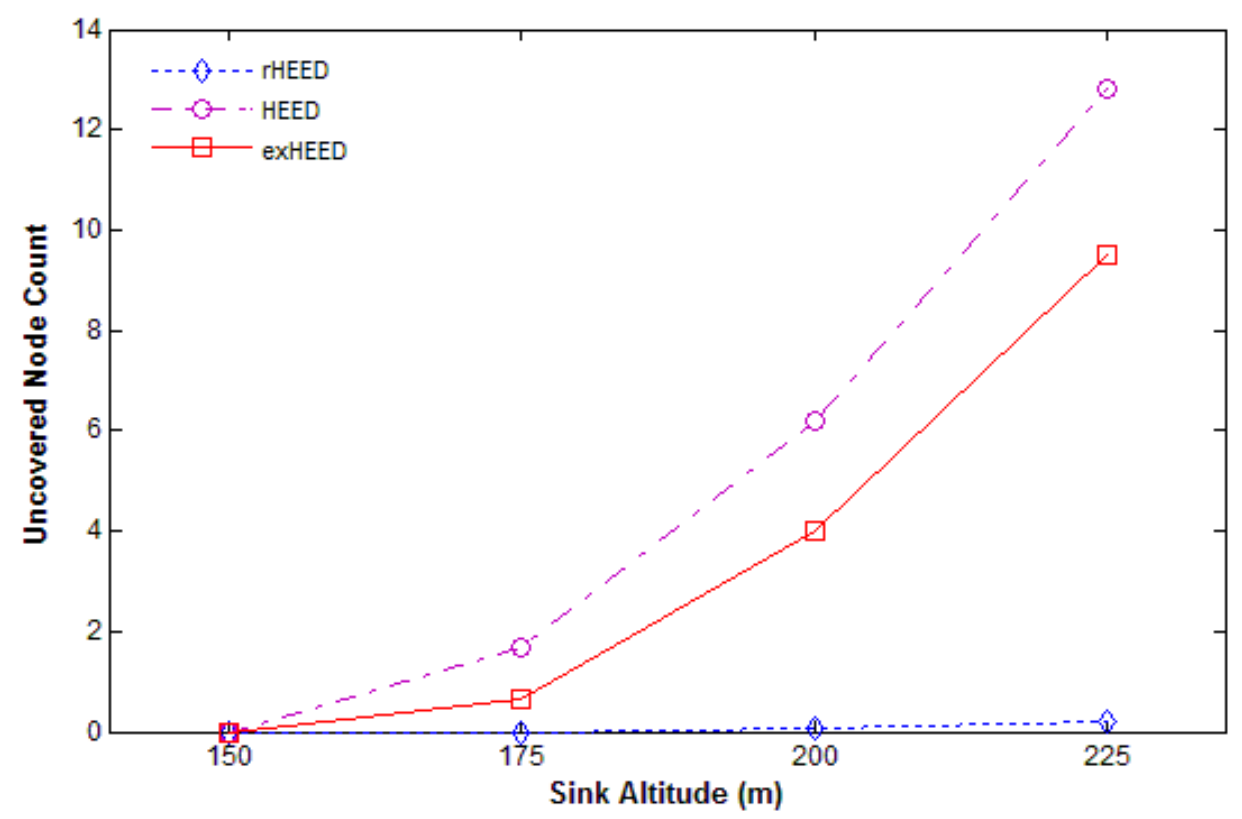

Figure 7 The Effect of the Mobile Sink Altitude on Coverage 
Clustering approach reduces the number of uncovered nodes within the network and increases the connectivity. Fig. 7 shows the number of uncovered nodes within the network when the clustering algorithms applied in the WSN. HEED and ExHEED algorithms suffer from high altitudes of the sink node. It is seen in the Fig. 7 that number of uncovered nodes increases as the sink altitude gets higher than 150m for HEED and ExHEED. rHEED does not suffer such a problem. Clustering algorithm in rHEED forms the clusters considering the UAV path. Therefore, nodes get connected to the UAV indirectly with the use of UAV connected CHs. Direct members of the CHs access to the UAV in two hops. On the other hand, if few nodes remain uncovered, which is rare in rHEED, these nodes get connected to nearest sensor node to become a member of the cluster of that neighbor node. Therefore, clustering algorithm and multi-hop membership in rHEED avoids uncovered node problem totally.

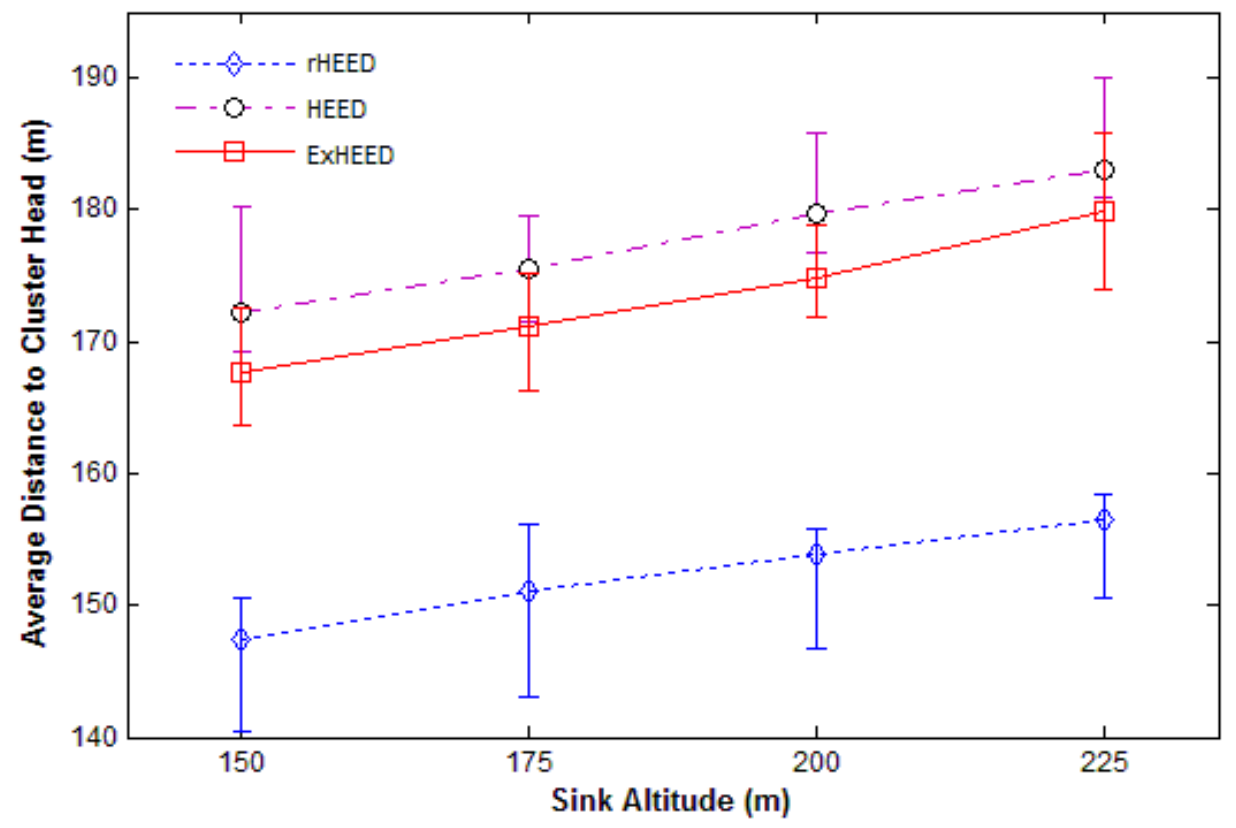

Figure 8 The Effect of the Mobile Sink Altitude on Distance to $\mathrm{CH}$.

Compared to the other approaches, rHEED has an advantage of utilizing distance proximity in the selection of $\mathrm{CH}$ using the RSSI value of $\mathrm{CH}$ in finalization phase of the algorithm. HEED and ExHEED methods choose $\mathrm{CH}$ that has high degree of neighborhood and do not consider distance to $\mathrm{CH}$. Therefore, connectivity is higher in rHEED compared to the other approaches. The use of RSSI in clustering has another favorable outcome. It provides the nodes get connected to the closer CHs. Therefore, it helps reducing the average distance of member nodes to the $\mathrm{CH}$.

Fig. 8 shows average distance to $\mathrm{CH}$ for nodes in the network. Nodes in rHEED network are closer to their respective $\mathrm{CH}$ than HEED and ExHEED. This will lead to lower transmission power levels and lower energy consumption in rHEED network. Moreover, lower transmission ranges to the sink node reduces the failures on transmissions due to the link quality. Side effects of the interference are reduced in rHEED compared to the other approaches for these reasons.

Table 5 Comparison of Cluster Related Values after Clustering

\begin{tabular}{|l|c|c|}
\hline \multicolumn{1}{|c|}{ Protocol } & $\begin{array}{c}\text { Average Single Cluster } \\
\text { Head Count }\end{array}$ & Cluster Count \\
\hline HEED & 11.59 & 40.11 \\
\hline ExHEED & 8.25 & 37.52 \\
\hline rHEED & 0.58 & 30.6 \\
\hline
\end{tabular}


Table 5 shows cluster related results that affect the performance metrics which are obtained at the end of the clustering process. The proposed approach, rHEED, has the minimum cluster count and single $\mathrm{CHs}$ count values. There is not any uncovered nodes rHEED, which affects the $\mathrm{CH}$ count. ExHEED has lower clusters and single $\mathrm{CH}$ count than HEED due to the core algorithm that it runs in the clustering process. ExHEED selects some extra $\mathrm{CH}$ among uncovered nodes instead of electing all uncovered node as $\mathrm{CH}$ which is the case in HEED.

\subsection{Comparison of Energy Consumption Levels}

During data gathering phase, energy consumption on transmissions between the sensor nodes and the $\mathrm{CHs}$ and transmissions between the $\mathrm{CHs}$ and $\mathrm{UAV}$ are affected from the distances between sensor nodes and $\mathrm{CHs}$ and from the distances (depending on the altitude of the UAV) between CHs and the UAV due to the transmission power levels. Fig. 9 shows that rHEED has superiority over the HEED and ExHEED. Nodes in rHEED consume much less energy compared to the others approaches. rHEED selects nodes as $\mathrm{CH}$ according to proximity to sink trajectory. Therefore, CHs in rHEED transmit with lower power levels to the UAV compared to the other protocols. We note that at higher altitudes, energy consumption does not linearly increase due to the coverage reduction over WSNs. As the altitude increases, reduction in the coverage will decrease the number of nodes which receive periodic beacon messages sent by UAV. It should be remembered that the receiving current is higher than transmitting, so beacon messages have significant impact on energy consumptions. Coverage reduction helps to reduce system-wide energy consumption due to reduction on the number of nodes which consume power on receptions.

Energy expended during clustering period is considered as wasteful energy consumption [4] and has great impact on total energy consumption. Fig. 10 shows that HEED has the minimum energy consumption on clustering period which is very close to rHEED. ExHEED suffers from extra core extraction algorithm both before and after the iterations of protocol and this leads to extra message traffic during the clustering phase. Results for rHEED is very close to the results for HEED but is slightly higher than HEED. The reason is that in finalization phase of the rHEED algorithm, uncovered nodes select the nearest cluster member nodes as their first level parent nodes with the cost of extra one more message traffic than HEED. In this tradeoff, the gain is the less number of CHs in rHEED compared to the HEED as presented in Table 5.

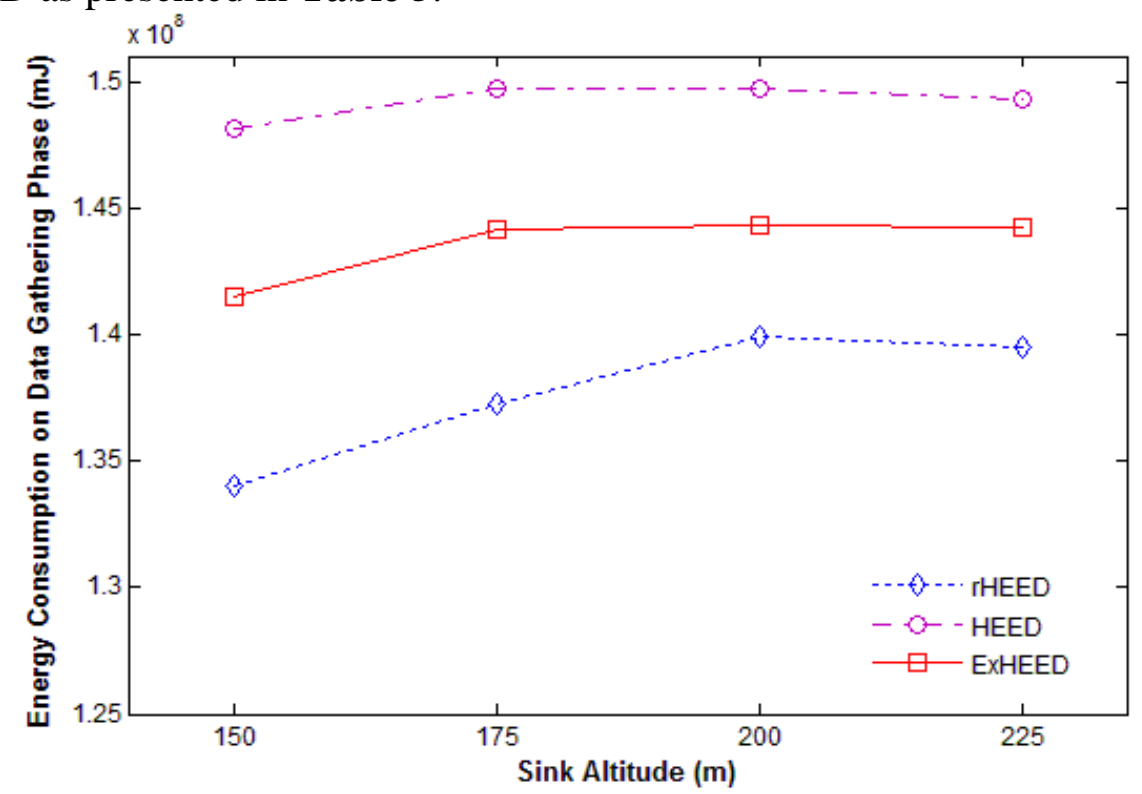

Figure 9 The Effect of the Mobile Sink Altitude on Energy Consumption during Data Gathering Phase. 


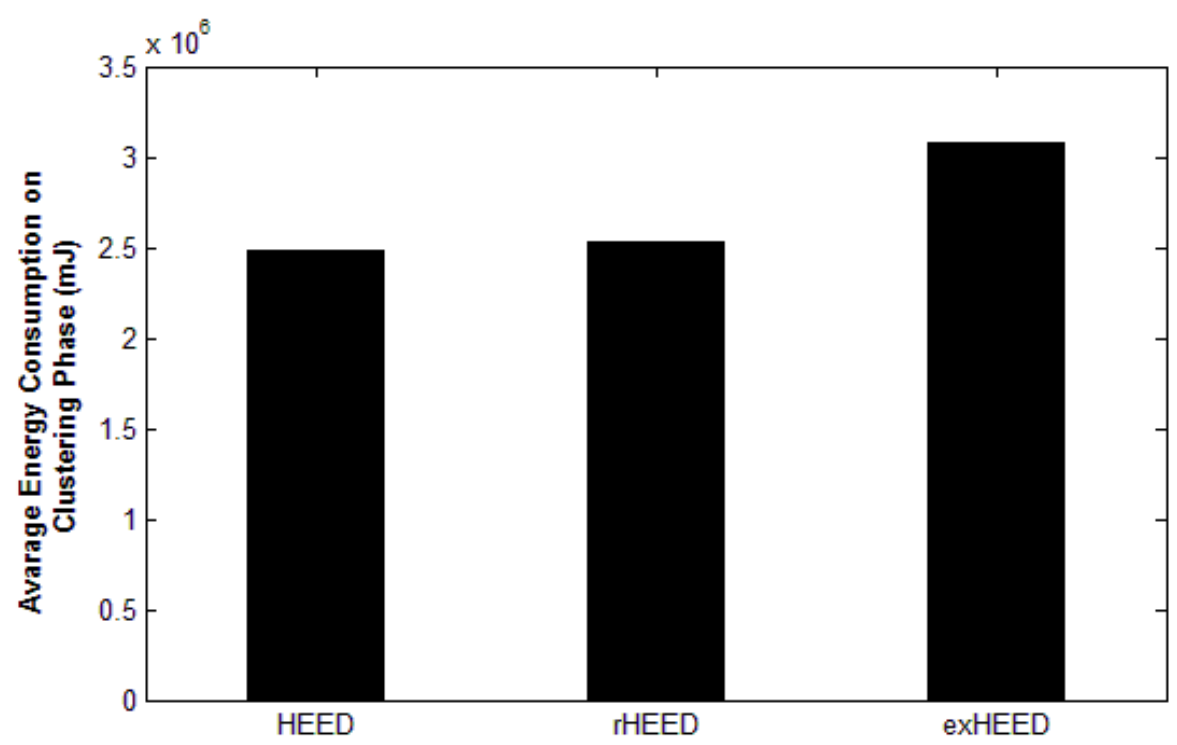

Figure 10 Average Energy Consumption on Clustering Phase.

Total energy consumption within the network is presented in Fig. 11. It is seen that total energy consumption in the system has similar results as in the energy consumption on data gathering shown in Fig. 9. The main difference is that the clustering energy load of ExHEED increases total energy consumption of this protocol. Another valuable outcome is that HEED still has the maximum total energy consumption although its clustering energy consumption is relatively lower than other approaches. HEED algorithm forms the clusters earlier than others with lower energy consumption, but it pays for it in the data gathering phase with the highest energy consumption. It can also be concluded that data gathering phase must be too long than clustering period, otherwise clustering energy consumption wastes the energy resources.

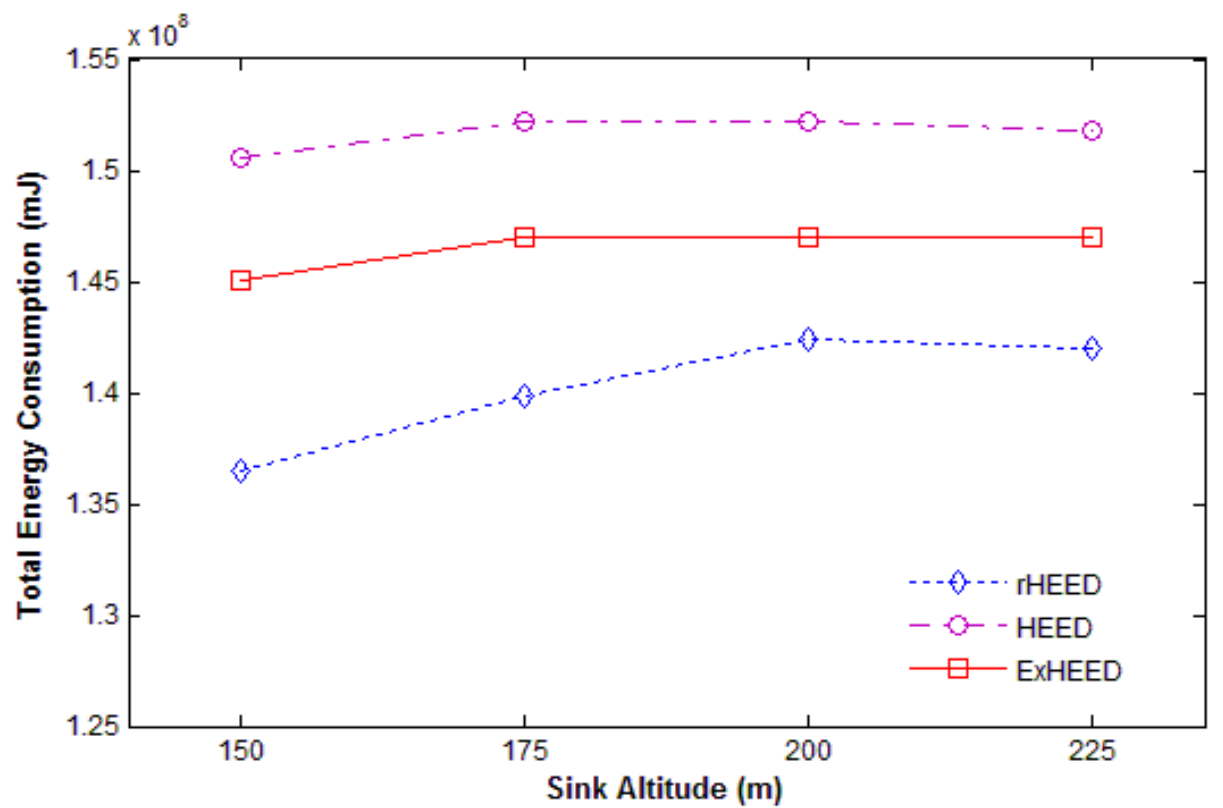

Figure 11 The Effect of the Mobile Sink Altitude on Total Energy Consumption.

\subsection{Deviations on the Results of Performance Metrics}

In this subsection, the deviations on the obtained results are examined. At each clustering phase, new $\mathrm{CHs}$ are selected. Therefore, number of $\mathrm{CHs}$ and distances to $\mathrm{CHs}$ vary at each clustering phase, which affects the performance of the networks. 


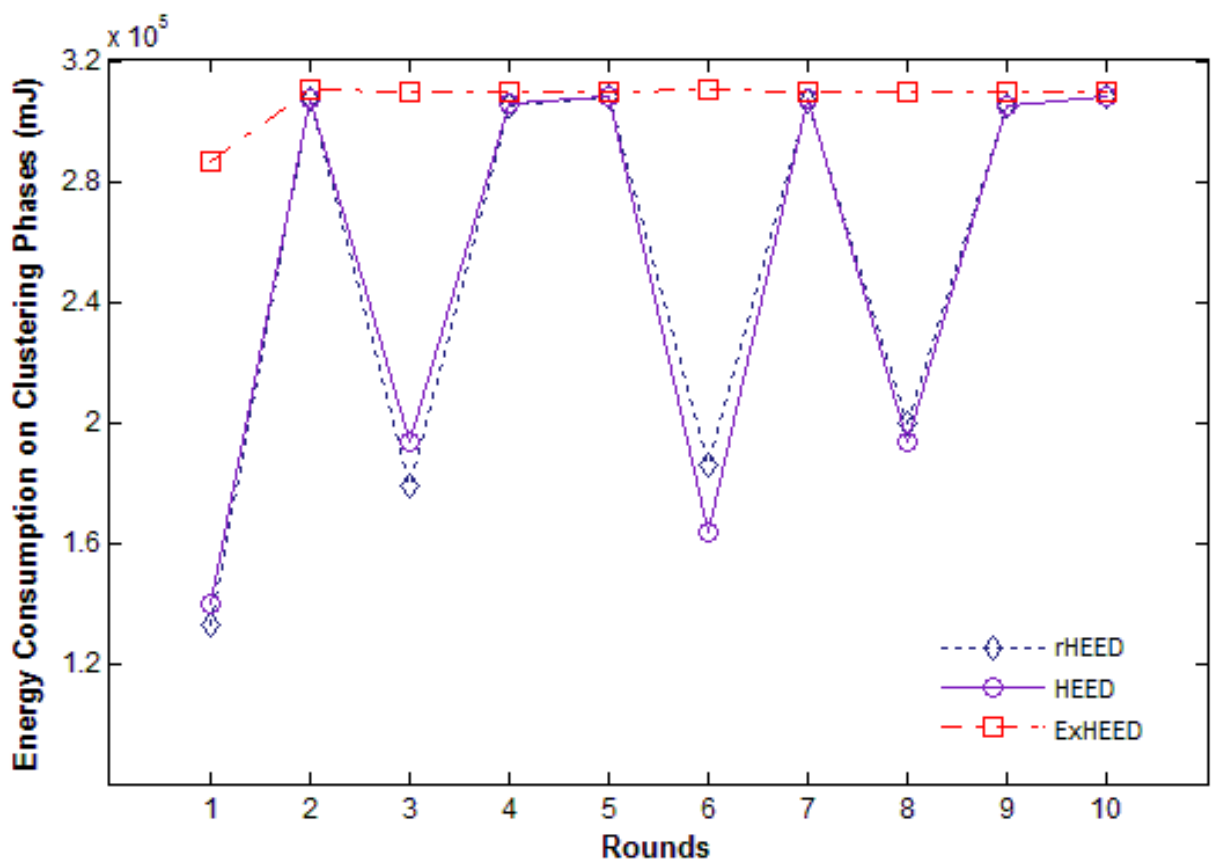

Figure 12 Energy Consumption on Clustering Phase at Each Round (Sink Altitude is 200m).

As shown in Fig. 12, clustering energy consumption in ExHEED is balanced, however it always shows relatively higher energy consumption in each round. ExHEED uses core extraction algorithm before and after the iterations, so its clustering energy consumption is high. Most of the time, some small set of sensors become core heads with core extraction message, which leads to balanced energy consumption in every round. On the other hand HEED and ExHEED select $\mathrm{CH}$ approximately from entire network. Thus deviation is high in both algorithms. However, they select more robust nodes from the entire candidate nodes.

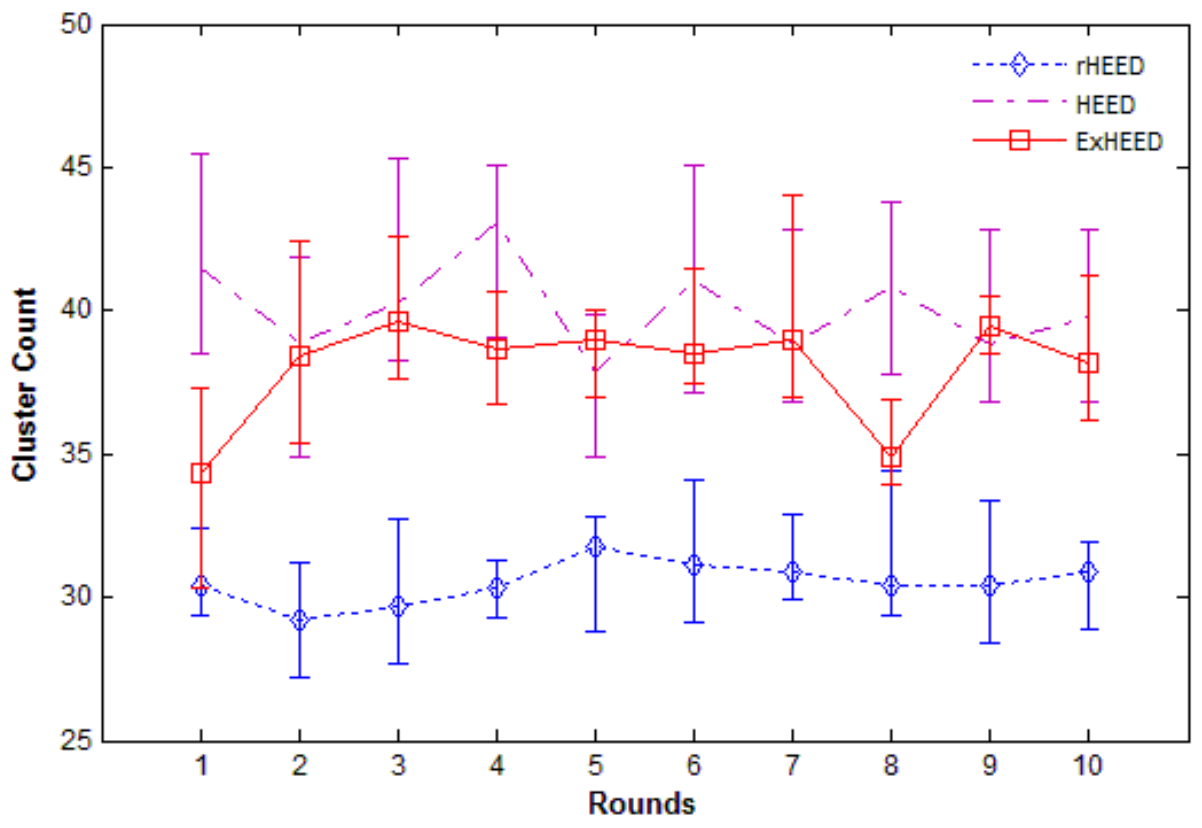

Figure 13 Cluster Count at Each Round (Sink Altitude is 200m)

Fig. 13 shows the number of clusters at each round. Cluster counts are stable in rHEED algorithm because rHEED avoid self (single) CHs to appear in the network. Therefore, rHEED has a little deviation on the cluster count. ExHEED shows relatively stable $\mathrm{CH}$ count compared to the HEED (but not to the rHEED) because of the core algorithm applied at the end of the iterations which reduces single cluster head count in ExHEED. 


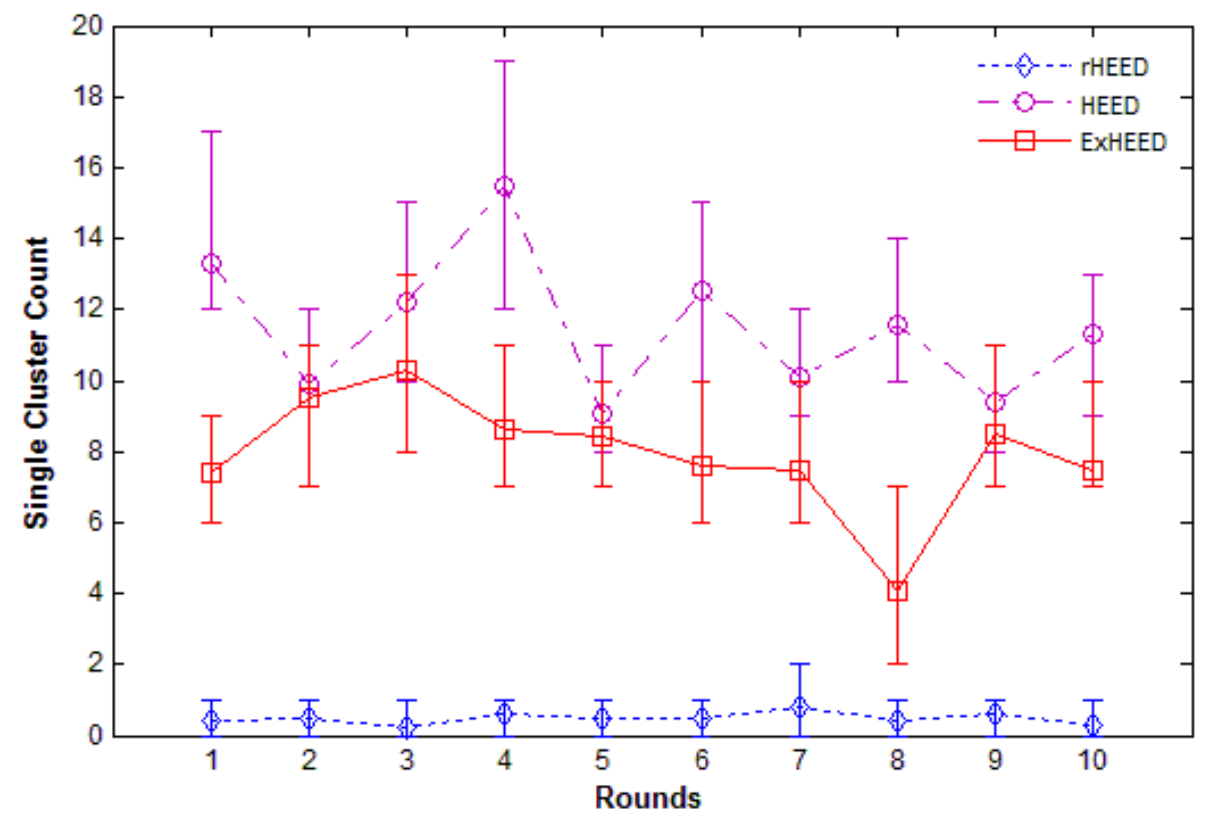

Figure 14 Single Cluster Count at Each Round (Sink Altitude is 200m)

Fig. 14 shows the number of single cluster head counts. To provide the load balancing in the network, single cluster head number should be low and unnecessary $\mathrm{CHs}$ should be avoided. Moreover, single clusters increase deviation of cluster sizes. In Fig. 14, it seen that HEED has the highest single cluster head due to uncovered nodes at the end of the iterations, which thereafter become CHs. ExHEED reduces single cluster head count with the applied core algorithm. rHEED avoids single cluster head with the applied algorithms in the proposed approach. The number never reaches to 0 because randomly distribution of nodes over the operation area places some nodes at positions where they have no neighbors within the communication range.

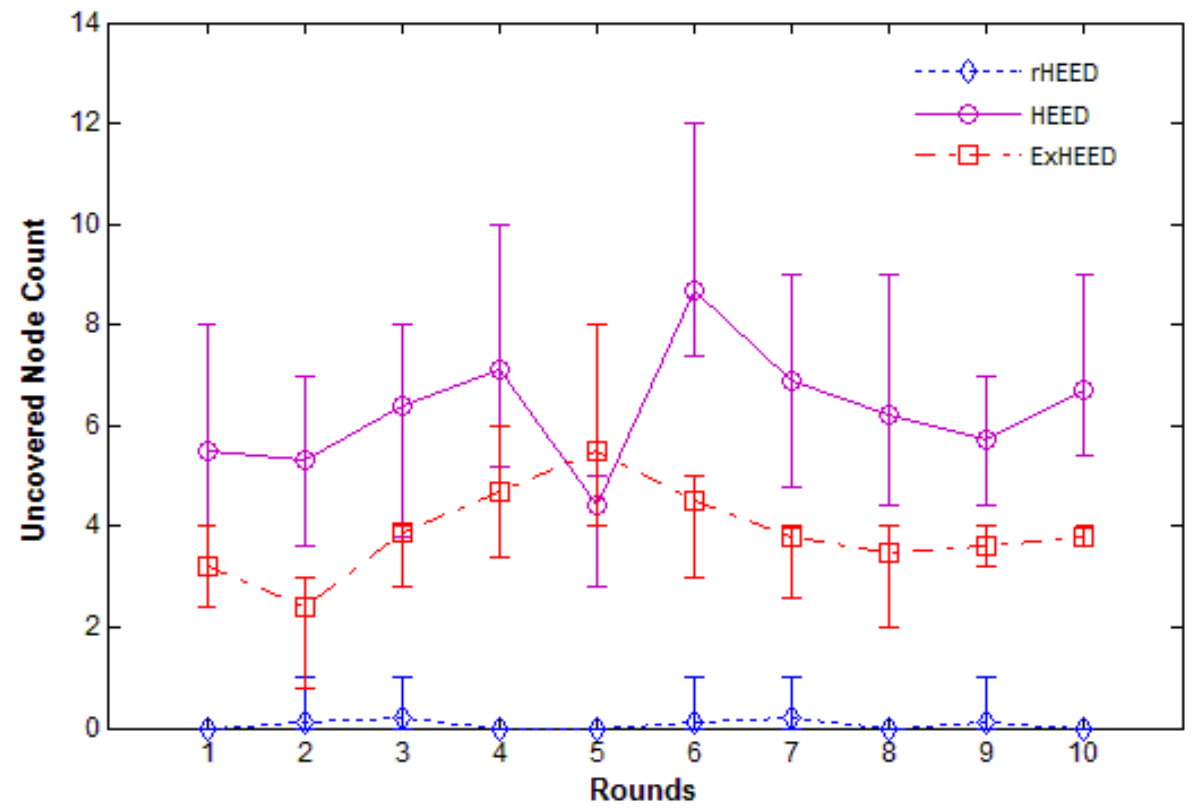

Figure 15 Uncovered Node Count at Each Round (Sink Altitude is 200m)

In Fig. 15, uncovered nodes that have no connection with a $\mathrm{CH}$ or UAV are examined. rHEED, as mentioned previously, increases connectivity. On the other hand, at different rounds HEED and ExHEED select CHs from different locations. This leads to uncovered node count in different values. 
As seen in Fig.13-15, there are high variations in the number of clusters, single-clusters and uncovered sensor nodes respectively for the HEED and ExHEED algorithms. HEED algorithm allows single clusters (cluster with a $\mathrm{CH}$ but no members) to be constructed. ExHEED has a method to reduce the number of single clusters, but it does not work efficiently. Possibility of construction of single clusters with the use of different seeds at each simulation run causes high variations on the measurements for HEED and ExHEED. It appears as a problem for such kind of clustering algorithms. However, rHEED, the proposed approach is not affected and does perform similarly at each simulation run. rHEED algorithm is more stable with respect to these algorithms. The small deviations in rHEED are due the previously described reason that randomly distribution of nodes over the operation area places some nodes at positions where they have no neighbors. These deviations are very small and can be considered as negligible for rHEED.

\section{Conclusions}

Large number of sensor node deployment over a geographical area (e.g. inaccessible areas) imposes some constraints on data transfer to the sink in WSNs. The use of static sinks is not practical due to energy-related constraints and end-to-end reliability requirements. Connectivity problems caused by random node deployment, noisy channels, harsh environments and link failures exacerbate this problem. The use of mobile sinks (e.g. UAVs) is an effective solution method for the retrieval of sensor data in inaccessible locations and in large scale WSNs. In this study, we point out that some nodes within the network may remain uncovered depending on the path and altitude of the UAV and the radio in use. Due to the low-cost sensors which operate with low-data-rate, short range radios, the UAV has to fly at an altitude within sensor range. Therefore, some nodes remain uncovered depending on the path and altitude of the UAV. Our analysis suggests that clustering in such environments is a feasible approach to increase the coverage. Selection of cluster heads considering the path of the UAV becomes the most important issue in the clustering approach. Based on our analysis on coverage, we propose a dynamic and distributed clustering approach, namely rHEED, to reduce the energy consumption on clustering and communications to extend the lifetime of the network. Compared to the approaches in the literature, further aspects of the problem are considered in the formation of clusters and selection of cluster heads. Proposed approach uses the RSSI values received from UAV and the remaining energy levels of the nodes for the selection of cluster head. Member nodes of the clusters are also selected considering the RSSI values of the cluster heads. Proposed approach provides more stable and well-balanced clusters where single node clusters are avoided. We evaluate the proposed approach with the use of realistic path loss, MAC and PHY layer models with the help of MIXIM Framework. It is shown that proposed approach, rHEED, reduces the energy consumption while avoiding unnecessary cluster head formations and avoiding uncovered nodes in the network. This study has contributions to the energy efficiency requirements of WSNs applications where mobile sink is used. This study is one of the first studies that considers UAV path in the formation of clusters.

\section{References}

[1]Akyildiz, I.F., Su,W., Sankarasubramaniam, Y., Cayirci, E., "A Survey on Sensor Networks," IEEE Communications Magazine, pages: 102-114, Aug 2002.

[2]Estrin, D., Girod, L., Pottie, G., Srivastavay, M., "Instrumenting The World With Wireless Sensor Networks", International Conference on Acoustics, Speech, and Signal Processing, pages: 2033-2036, May 2001.

[3]Yoneki, E., Bacon, J., “A Survey of Wireless Sensor Network Technologies: Research 
Trends And Middleware's Role", Technical Report, Number 646, Computer Laboratory, UCAM-CL-TR-646, ISSN 1476-2986, Sept. 2005.

[4]Younis, O., Fahmy, S., "Distributed Clustering in Ad-hoc Sensor Networks: A Hybrid, Energy-Efficient Approach", Proceedings of IEEE INFOCOM, Mar. 2004.

[5]Anastasi, G., Conti, M., Di Francesco, M.," Data collection in sensor networks with data mules: An integrated simulation analysis", IEEE Symposium on Computers and Communications (ISCC 2008), page: 1096 - 1102, Jul. 2008.

[6]Zhu, X., Zhang, W., "A Mobile Agent-based Clustering Data Fusion Algorithm in WSN", International Journal of Electrical and Computer Engineering, Volume: 5, Issue: 5, pages: 277-280, 2010.

[7]IEEE Computer Society, "IEEE Std.802.15.4-2006, Wireless Medium Access Control (MAC) and Physical Layer (PHY) Specifications for Low-Rate Wireless Personal Area Networks (WPANs)", Sep. 2006.

[8]Texas Instruments TI CC2420 2.4 GHz IEEE 802.15.4/ZigBee-ready RF Transceiver Datasheet, Url < http://www.ti.com/product/cc2420 >, accessed at 03.11.2011.

[9] Polastre, J., Szewczyk, R., Culler, D., "Telos: Enabling Ultra-low Power Wireless Research", Fourth International Symposium on Information Processing in Sensor Networks, Pages: 364 - 369, Apr. 2005.

[10]Telos Ultra low power IEEE 802.15.4 compliant wireless sensor module, Revision B : Humidity, Light, and Temperature sensors with USB Datasheet. Url < http://www2.ece.ohio-state.edu/ bibyk/ee582/ telosMote.pdf >, accessed at 15.10.2011.

[11]Heinzelman, W.R., Chandrakasan, A., Balakrishnan, H., "Energy-Efficient Communication Protocol for Wireless Microsensor Networks," Proceedings of the 33rd International Conference on System Sciences (HICSS '00), Jan.2000.

[12]Heinzelman, W.R., Chandrakasan, A., Balakrishnan, H., "An Application-Specific Protocol Architecture for Wireless Microsensor Networks" IEEE Transactions on Wireless Communications, Volume: 1, Issue: 4, pages: 660-670, Oct. 2002.

[13]Bandyopadhyay, S., Coyle, E.J., "An Energy Efficient Hierarchical Clustering Algorithm for Wireless Sensor Networks", INFOCOM Twenty-Second Annual Joint Conference of the IEEE Computer and Communications, Volume: 3, pages: 1713-1723, Apr. 2003.

[14]Huang, H., Wu, J., "A Probabilistic Clustering Algorithm in Wireless Sensor Networks", Proceeding of IEEE 62nd Semiannual Vehicular Technology Conference (VTC), Volume: 3, pages: 1796-1798, Sept. 2005.

[15]Sinha, P., Sivakumar, R., Bharghavan, V., "CEDAR: a Core-Extraction Distributed Ad hoc Routing Algorithm", IEEE Journal on Selected Areas in Communications, Volume: 17, Issue: 8, pages: 1454 - 1465, Aug 1999.

[16]Ping Ding, Joanne Holliday, Aslıhan Çelik, "Distributed Energy-Efficient Hierarchical Clustering for Wireless Sensor Networks", Proceedings of the IEEE International Conference on Distributed Computing in Sensor Systems, pages: 322-339, Jun. 2005

[17]Fazackerley, S., Paeth, A., Lawrence, R., "Cluster Head Selection Using RF Signal Strength", Proceedings of Canadian Conference on Electrical and Computer Engineering (CCECE'09), pages: 334 - 338, May 2009.

[18]Pantziou, G., Mpitziopoulos, A., Gavalas, D., Konstantopoulos, C., Mamalis, B., 
"Mobile Sinks for Information Retrieval from Cluster-Based WSN Islands", Proceedings of the 8th International Conference on Ad-Hoc, Mobile and Wireless Networks (ADHOC-NOW '09), Springer-Verlag, pages: 213-226, Sept. 2009.

[19]Lotfinezhad, M., Liang, B., "Energy Efficient Clustering in Sensor Networks with Mobile Agents", Proceedings of the IEEE Wireless Communications and Networking Conference (WCNC'05), Volume: 3, pages: 1872-1877, Mar. 2005.

[20]Friis, H.T., "A Note on a Simple Transmission Formula", Proceedings of the IRE, Volume: 34 , Issue: 5, pages: 254 - 256, May 1946.

[21]Blace, R.E., Eltoweissy, M., Abd-Almageed, W., "Threat-Aware Clustering in Wireless Sensor Networks", IFIP Advances in Information and Communication Technology (AICT), Volume: 264, Issue:264, pages: 1 - 12, 2011.

[22]Sugar, R., Imre, S., "Adaptive Clustering Using Mobile Agents in Wireless Ad-Hoc Networks", in Proc. of the 8th International Workshop on Interactive Distributed Multimedia Systems, pages: 199-204, 2001.

[23]Denko, M., K., "The use of mobile agents for clustering in mobile ad hoc networks", in Proc. of the conference of the South African institute of computer scientists and information technologists on Enablement through technology, pages: 241-247, 2003.

[24]Morsly, Y., Aouf, N., Djouadi, M. "Dynamic decentralized/centralized free conflict UAV's team allocation", IEEE International Instrumentation and Measurement Technology Conference (I2MTC), Pages: 2340 - 2345, May 2012

[25]Bekmezci, İ., Sahingoz, Ö., K., Temel, Ş., "Flying Ad-Hoc Networks (FANET): A Survey", Ad Hoc Networks, Volume: 11, Issue: 3, Pages: 1254-1270, Jan 2013.

[26]The Wasp Micro Air Vehicle (MAV) Datasheet, Url < http://www.avinc.com/uas/small_uas/wasp >, accessed at 20.11.2011.

[27]U.S. Air Force Fact Sheet, RQ-11B Raven System, Url < http://www.avinc.com/uas/small_uas/raven >, accessed at 20.11.2011.

[28]Bayraktar Mini-UAV Datasheet, Url < http://www.baykarmakina.com/en/MiniUAV >, accessed at 20.11.2011.

[29]Puma AE Datasheet, Url < http://www.avinc.com/uas/small_uas/puma >, accessed at 20.11.2011.

[30]Falco UAV System, Selex Galileo. Url<http://www.selexgalileo.com/ SelexGalileo/EN/Business/Products/Unmanned_Aerial_Systems/index.sdo >, accessed at 20.11.2011.

[31]Url < http://www.omnetpp.org/ >, accessed at 08.10.2011.

[32]Url < http://mixim.sourceforge.net/ >, accessed at 10.10.2011.

[33]Köpke, A., Swigulski, M., Wessel, K., Willkomm, D. Haneveld, P.T.K., Parker, T.E.V., Visser, O.W., Lichte H.S., Valentin S., "Simulating Wireless and Mobile Networks in OMNeT++: The MiXiM Vision", Proceedings of the 1st International Conference on Simulation Tools and Techniques for Communications, Networks and Systems \& Workshops (Simutools '08), Mar. 2008. 
Huseyin Okcu received the B.Sc. degree in Electrical and Electronics Engineering Department from Naval Academy, Istanbul, Turkey, in 2003. He received his M.Sc. degree in C4I Program from Naval Science and Engineering Institute, Istanbul, Turkey, in 2012. He is currently working at Turkish Naval Academy (TNA) in Istanbul, Turkey. His research interests include Wireless Sensor Networks and Mobile Communications.

Mujdat Soyturk received the B.Sc. degree in Industrial Engineering from Naval Academy, Istanbul, Turkey, in 1994. He received his M.Sc. and Ph.D. degrees both from Istanbul Technical University, Istanbul, Turkey, in Computer Engineering in 2002 and 2007, respectively. He was a visiting researcher for C4I Systems (Command, Control, Computer, Communications and Intelligence Systems) in Naval Postgraduate School (NPS), Monterey, CA, in 2009. His research interests include Wireless and Mobile Communications, Sensor and Ad Hoc Networks, Vehicular Networks, and Net-Centric Communications.

Please note that this version of the paper is the post-peer review, accepted paper submitted for final publication. The printed and reformatted version is accessible at the original source of the publication (http://www.inderscience.com/info/inarticle.php?artid=59912), where cited as "Huseyin Okcu, Mujdat Soyturk. Distributed Clustering Approach for UAV Integrated Wireless Sensor Networks, International Journal of Ad Hoc and Ubiquitous Computing, Vol.15, No. 1-3, pp.106-120, 2014 (2014)". 